Draft Version February 14, 2018

Preprint typeset using $\mathrm{LAT}_{\mathrm{E}} \mathrm{X}$ style emulateapj v. 5/2/11

\title{
HUBBLE SPACE TELESCOPE/ADVANCED CAMERA FOR SURVEYS CONFIRMATION OF THE DARK SUBSTRUCTURE IN A5201
}

\author{
M. J. JEE ${ }^{2}$, H.. HOEKSTRA ${ }^{3}$, A. MAHDAVI ${ }^{4}$, AND A. BABUL ${ }^{5,6}$ \\ Draft version February 14, 2018
}

\begin{abstract}
We present the results from a weak gravitational lensing study of the merging cluster A520 based on the analysis of Hubble Space Telescope/Advanced Camera for Surveys (ACS) data. The excellent data quality allows us to reach a mean number density of source galaxies of 109 per sq. arcmin, which improves both resolution and significance of the mass reconstruction compared to a previous study based on Wide Field Planetary Camera 2 (WFPC2) images. We take care in removing instrumental effects such as the trailing of charge due to radiation damage of the ACS detector and the positiondependent point spread function (PSF). This new ACS analysis confirms the previous claims that a substantial amount of dark mass is present between two luminous subclusters. We examine the distribution of cluster galaxies and observe very little light at this location. We find that the centroid of the dark peak in the current ACS analysis is offset to the southwest by $\sim 1^{\prime}$ with respect to the centroid from the WFPC2 analysis. Interestingly, this new centroid is in better spatial agreement with the location where the X-ray emission is strongest, and the mass-to-light ratio estimated with this centroid is much higher $\left(813 \pm 78 M_{\odot} / L_{R \odot}\right)$ than the previous value; the aperture mass based on the WFPC2 centroid provides a slightly lower, but consistent mass. Although we cannot provide a definite explanation for the presence of the dark peak, we discuss a revised scenario, wherein dark matter with a more conventional range $\left(\sigma_{D M} / m_{D M}<1 \mathrm{~cm}^{2} \mathrm{~g}^{-1}\right)$ of self-interacting cross-section can lead to the detection of this dark substructure. If supported by detailed numerical simulations, this hypothesis opens up the possibility that the A520 system can be used to establish a lower limit of the self-interacting cross-section of dark matter.

Subject headings: gravitational lensing — dark matter - cosmology: observations - X-rays: galaxies: clusters — galaxies: clusters: individual (Abell 520) — galaxies: high-redshift
\end{abstract}

\section{INTRODUCTION}

Galaxy clusters are comprised of dark matter, cluster galaxies, and hot plasma. When two clusters collide, it is believed that the galaxies and dark matter temporarily dissociate from the hot plasma because the latter is collisional and subject to ram pressure. Eventually, the dark matter of the cluster, the gravitationally dominant component, pulls the hot plasma back into its potential well, and the dissociation disappears. Therefore, in general we have a narrow time-window (a few Gyrs after the core pass-through) to witness observationally significant offsets between collisional and collisionless constituents of the clusters.

Detailed studies of these "dissociative" mergers (Dawson 2012) provide unique opportunities to enhance not only our astrophysical understanding of the cluster formation and evolution, but also our understanding of fundamental physics on the nature of dark matter. Because

\footnotetext{
1 Based on observations made with the NASA/ESA Hubble Space Telescope, obtained at the Space Telescope Science Institute, which is operated by the Association of Universities for Research in Astronomy, Inc.

2 Department of Physics, University of California, Davis, One Shields Avenue, Davis, CA 95616, USA

${ }^{3}$ Leiden Observatory, Leiden University, Leiden, The Netherlands

${ }^{4}$ Department of Physics and Astronomy, San Francisco State University, San Francisco, CA 94131, USA

${ }^{5}$ Department of Physics and Astronomy, University of Victoria, Victoria, BC, Canada

${ }^{6}$ Kavli Institute for Theoretical Physics, Kohn Hall, University of California, Santa Barbara, CA 93106, USA
}

a direct lab detection of dark matter particles may not happen within the current decade, these merging clusters with large offsets among the different cluster constituents are receiving growing attention (e.g., Springel \& Farrar 2007; Randall et al. 2008; Dawson 2012).

To date, only a few merging systems are known to possess such large dissociative features (e.g., Dawson et al. 2012, Merten et al. 2011, Bradac et al. 2008; Okabe \& Umetsu 2008; Soucail 2012), and only two systems, namely 1E0657-56 at $z=0.3$ (Markevitch et al. 2002; hereafter the "Bullet Cluster") and A520 at $z=0.2$ (Markevitch et al. 2005), are known to possess prominent X-ray "bow-shock" features, which serve as the definite evidence for a recent high-speed collision and allow us to estimate the relatively stable transverse velocity of the collision. Clowe et al. (2006) showed that the mass distribution of the Bullet Cluster revealed by weak-lensing closely follows the cluster galaxies, which themselves are offset from the X-ray emitting gas. The observation is claimed as proof of dark matter, and some studies (e.g., Markevitch et al. 2004; Randall et al. 2008) use the result to constrain an upper limit of the dark matter self-interaction cross-section.

Mahdavi et al. (2007; hereafter M07) reported a more puzzling case based on their weak-lensing analysis of A520 with Canada France Hawaii Telescope (CFHT) and Subaru images. The mass distribution of M07 generally follows the cluster galaxies as seen in the Bullet Cluster. However, what separates A520 from the Bullet Cluster is the presence of a "dark core", which coincides with 
the location of the peak of the X-ray emission. The dark core region does not contain any luminous cluster galaxies unlike the other mass peaks in M07. Although the initial independent analysis of A520 with Subaru images by Okabe \& Umetsu (2008) did not support the presence of the dark core, their final study carried out with the subset of their data (taken with the auto guider of the telescope turned on) agrees with M07 in that their weak lensing data shows the dark peak near the peak of the X-ray emission. This study of Okabe \& Umetsu (2008) serves as a good example to illustrate that the investigation of cluster substructures is prone to instrumental systematics such as imperfect point spread function models.

A logical extension of these studies is a follow-up investigation with space-based images, which provide a much higher density of source galaxies. If the dark core had appeared as an "unfortunate statistical fluke" due to fortuitous alignment of source galaxies, mass reconstruction with a much higher source density must reduce the probability of this chance alignment and thus the significance of the feature. In Jee et al. (2012; hereafter J12), we presented a Hubble Space Telescope (HST) Wide Field Planetary Camera 2 (WFPC2) weak lensing analysis of A520 and confirmed the results of M07 and Okabe \& Umetsu (2008). Both the two-dimensional mass map and the aperture mass results of J12 are consistent with M07, except for the detection of two additional mass peaks, which were not reported in M07. One of the two new peaks (labeled as P5 in J12) resolves one of the questions raised in M07, who considered the absence of any significant mass peak around this location also discordant with our common light-traces-mass hypothesis along with the presence of the significant mass in the dark core region. The other new peak (labeled as P6 in J12) is detected $\sim 200 \mathrm{kpc}$ south of the dark core and coincides with the spatial distribution of the cluster galaxies. As for the dark core both J12 and M07 discuss a number of possibilities that may lead to the observation, but neither study could exclusively single out one definite scenario responsible for these observations.

The scenarios considered in J12 and M07 are: 1) a possible presence of a background high-redshift $(z>1)$ cluster at the location of the dark peak, 2) a rejection of cluster galaxies during a complex multi-body collision, 3 ) a filament elongated along the line-of-sight direction, 4) self-interacting dark matter, and 5) a compact high $\mathrm{M} / \mathrm{L}$ group. We reject the first scenario because our spectroscopic survey data do not indicate the presence of a possible $z>1$ cluster at the dark peak, the Xray emission line is consistent with the A520 redshift, and the dark peak is seen in ground-based weak-lensing, where the mean source redshift is lower than $z \sim 1$. Scenario 2 is discarded because no numerical simulations have shown that brightest cluster galaxies are ejected during three-body encounter, although it can happen to faint satellite galaxies. The third possibility is unlikely because the filament must be very thin in such a way that most of the projected mass is confined to the central $r<150 \mathrm{kpc}$ region around the dark peak. The fourth scenario is highly disfavored because the anticipated collisional cross-section of dark matter by M07 is much higher than the values estimated from the Bullet Cluster. The last scenario has not been completely ruled out yet. However, the implied $\mathrm{M} / \mathrm{L}$ value of the group is too high to be bracketed by the values found in the literature.

The improved sampling resolution and sensitivity of the Advanced Camera for Surveys (ACS) allow for a more efficient coverage of A520 reaching a higher source density in principle. Recently, Clowe et al. (2012; hereafter $\mathrm{C} 12$ ) presented the results from their weak lensing analysis of A520, this time using such ACS data. They reported that no such dark peak is seen in their analysis. The rest of the weak lensing substructures of C12 closely resemble those of J12 including the aforementioned two new mass peaks of J12. Although this claim of $\mathrm{C} 12$, if true, may resolve the "mystery" reported by J12, M07, and Okabe \& Umetsu (2008), the discrepancy raises a different kind of puzzle, namely why is the result of the C12 weak lensing study so different from those of three previous independent studies.

To address this issue, we perform an independent weak lensing analysis of A520 using the same ACS data used by $\mathrm{C} 12$. We provide a detailed comparison of the results with those from C12. The structure of the paper is as follows. In $\S 2$ we present the data and our analysis. Correction of instrumental effects is detailed in $\S 3$. The mass reconstruction is discussed in $\S 4$ and mass estimates are presented in $\S 5$. We compare our results to those of $\mathrm{C} 12$ in $\S 6$ and examine whether the new observations can constrain the interaction crosssection of dark matter in $\S 6$. Throughout this paper we use $\left(\Omega_{M}, \Omega_{\Lambda}, h\right)=(0.3,0.7,0.7)$ for cosmology unless explicitly stated otherwise. This gives a plate scale of $\sim 3.3 \mathrm{kpc} /{ }^{\prime \prime}$ at the redshift $(z=0.2)$ of Abell 520. All the quoted uncertainties are at the 1- $\sigma(\sim 68 \%)$ level.

\section{DATA}

We retrieved the HST/ACS images of A520 (PI: Clowe) from the Mikulski Archive for Space Telescopes (MAST) $\sqrt{7}$ in 2012 July after the new charge transfer inefficiency (CTI) correction of Ubeda and Anderson (2012) had become available. The cluster was observed in the Cycle 18 (2011 February and April) and the raw data are severely affected by CTI. The images are comprised of four pointings in F435W, F606W, and $\mathrm{F} 814 \mathrm{~W}$ to cover the approximately $7^{\prime} \times 7^{\prime}$ central region of the cluster. The exposure time per pointing is $4,600 \mathrm{~s}$ for $\mathrm{F} 814 \mathrm{~W}$ whereas it is a factor of two smaller for both F435W and F606W. A similar exposure time per pointing $(4,400 \mathrm{~s})$ was used for the WFPC2 observation (PI: Dalcanton) of A520, although the ACS image is in general 1.4 magnitude deeper because of its higher (more than a factor of 3) sensitivity. However, around the dark core the WFPC2 image provides comparable depth because the WFPC2 observation was designed in such a way that the footprints overlap substantially in that region, although the ACS weak lensing analysis benefits from the improved sampling. We use the F814W data as the primary data set for the lensing analysis. However, we verify that a parallel weak-lensing analysis with the $\mathrm{F} 606 \mathrm{~W}$ image gives a consistent result yet with slightly increased noise because of the shallower depth.

\footnotetext{
${ }^{7}$ http://archive.stsci.edu

8 CTI causes systematic elongation of object shapes along the readout direction. Readers are referred to 3.1 for details.
} 
We combine the four ACS pointings into a single mosaic image, which is used to measure the galaxy shapes (note that we keep track of the combined PSF). As discussed in more detail in $\$ 2$, a careful treatment of the impact of the CTI is critical. To investigate this, we created three different versions of mosaic images. The first mosaic was generated with the FLT files processed by the CALACS pipeline (Hack et al. 2003). These images are corrected for the bias stripping noise 9 with no CTI correction applied. The second mosaic is made from the FLC files also processed by the CALACS pipeline, which removes both bias stripping noise and CTI trails using the latest 2012 CTI model of Ubeda and Anderson (2012). This latest model was not available to C12. Finally, for our third mosaic image, we use the PixCteCorr script to correct the CTI effects as per the 2009 CTI model (Anderson \& Bedin 2010). This old model is considered inferior to the latest model for the accuracy of the correction in the low-flux regime. We defer the detailed comparison to 33.1 .

The image offsets are computed by comparing the coordinates of common astronomical sources. This process can be easily automated for the image set observed at the same pointing with an accuracy of $\lesssim 0.01$ pixels. However, the offsets between different pointings are difficult to determine reliably using automated algorithms because the small overlapping areas ( 200 pixels) and the dense distribution of cosmic rays make only a few objects available for shift estimation. Therefore, we choose to create a separate stack for each pointing as an intermediate step and to use the catalog from the resulting image (where cosmic rays are removed and fainter sources are available) to determine accurate offsets between different pointings $(\sim 0.02 \mathrm{pixel})$. The final full $2 \times 2$ mosaic is generated by the MultiDrizzle software (Koekemoer et al. 2002) with the Lanczos3 drizzling kernel and an output pixel scale of $0 . \prime 05$. The Lanczos3 kernel closely approximates the theoretically ideal sinc $(\sin x / x)$ interpolation kernel by truncating the oscillation beyond the third pixel from the center. In Jee et al. (2007a), we demonstrate that this Lanczos3 drizzling kernel is superior to the "square" kernels in minimizing both aliasing and noise correlation and gives the sharpest PSF. We note that $\mathrm{C} 12$ used a square kernel with an output pixel scale of $0 . \prime 05$ to drizzle the ACS images, which may not be optimal for measuring accurate shapes of small galaxies.

\section{ANALYSIS}

The key ingredient in any weak lensing analysis is the accurate measurement of the shapes of the source galaxies. A number of observational effects prevent us from simply using the observed shapes. Instead we need to characterize these instrumental distortions and correct for them. In addition to the usual correction for the PSF (both size and anisotropy), the analysis of ACS data needs to account for the trailing of charge due to CTI. Since charge trapping happens every time charges are transferred from one pixel to another, CTI is greater for pixels farther from the readout register. After the trapped charges in one transfer are released, a fraction of them remain trapped during the next transfer. This

\footnotetext{
${ }^{9}$ http://www.stsci.edu/hst/acs/software/destripe/
}

cascading effect leads to trails, leading to a change in the shapes of galaxies. Both photometry and shape measurements are affected by CTI, although both are sensitive to different species of traps.

Although this undesirable artifact happens in every $\mathrm{CCD}$, it is an especially serious concern for space telescopes where the CCDs are subject to constant space radiation and the sky background is very low. The number of defects increases roughly linearly with time, and the A520 data taken in the Cycle 18 (2011 February and April) are severely affected by this CTI problem.

J12 discussed the potential impact of uncorrected CTI in the WFPC2 analysis. Although WFPC2 at the time of the observation of A520 was in orbit longer than ACS at the time of the current observation, the much smaller size of the readout distance ( 800 pixels vs. 2048 pixels) makes the overall impact less severe. In addition, the direction of the CTI trails differs for the three CCDs of WFPC2, which results in a more effective mixing of any residual CTI pattern.

\subsection{Correction for CTI}

We measure the CTI utilizing warm pixels and compact cosmic rays present in the same science data (Jee et al. 2009; Jee et al. 2011; J12). These sub-PSF features (hereafter SPFs) suffer from the charge transfer efficiency degradation but are not affected by the anisotropic PSF of the instrument. Hence we can single out CTI and perform a statistical analysis of their ellipticity as a function of charge transfer distance and flux.

We quantify the elongation of SPFs due to CTI using the ellipticity defined as

$$
\begin{gathered}
e=\frac{a-b}{a+b} \\
e_{+}=e \cos (2 \theta) \\
e_{\times}=e \sin (2 \theta)
\end{gathered}
$$

where $a$ and $b$ are the semi-major and -minor axis, respectively. $\theta$ is the orientation of the ellipse. For the current CTI measurement, we choose the serial readout direction as our $x$-axis and the parallel readout direction as our $y$-axsis.

The plot on the left panel of Figure 1 shows the $e_{+}$ component of the SPF ellipticity as a function of a charge transfer distance; because the $x$-axis is defined to be orthogonal to the readout direction, the sign of $e_{+}$becomes negative for CTI trails. Different colors represent different ranges of flux counts (after background being subtracted). Several features are worth noting in comparison with previous work. First, the CTI is still linear with transfer distance, as was observed in our previous studies (Jee et al. 2009; Jee et al. 2011; Hoekstra et al. 2011). Second, even the brightest SPFs are severely affected by CTI. Compared to our 2009 data, the slope of the SPFs at the flux range 2500-4000 $e^{-}$has increased by more than a factor of two, showing that the farthest $(\sim 2000$ pixels) SPFs suffer from a net ellipticity distortion of 0.2 whereas it was at the $\delta e \sim 0.1$ level for the data taken in the year 2009. Third, the flux-dependence of the slope is more complicated. In Jee et al. (2009; 2011), we observed that the CTI slope becomes more negative for decreasing flux until it sharply turns around at $\sim 300 e^{-}$ 

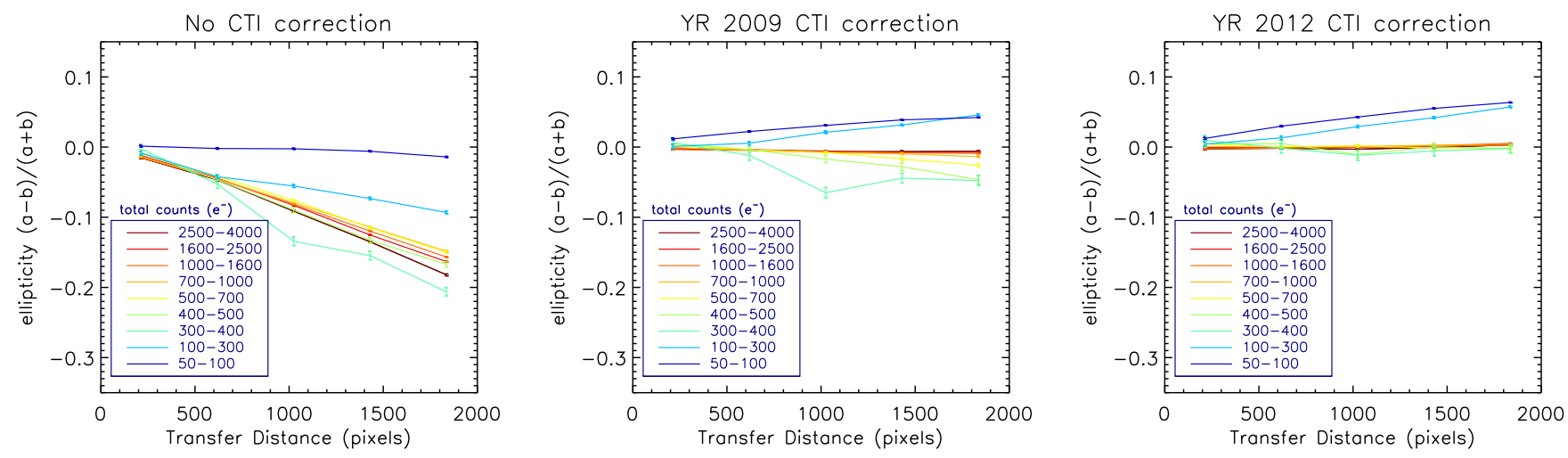

Figure 1. CTI effects in the A520 ACS data measured from the ellipticity of sub-PSF features (SPFs) such as cosmic rays and warm pixels. The counts are calculated with the background subtracted. The left plot shows the result from the uncorrected ACS images. Without any correction, severe charge trailings are present, and thus weak lensing based on the raw images will be non-negligibly biased. The middle panel displays the result when the old (Anderson \& Bedin 2010) pixel-based method is applied, which reduces the CTI artifacts. However, the residual ellipticities indicate that the results are not yet satisfactory. The right panel shows the result when the latest (Ubeda and Anderson 2012) correction is used. The latest correction method successfully takes care of these residual CTI effects for the SPFs with counts greater than $\sim 300$. Both corrections tend to overcorrect the CTI effects for low-count objects $(\sim 300)$. This might be because the current pixel-based method does not take into account the low-flux-limit CTI-mitigation reported in Jee et al. (2009).

(see Figure 31 of Jee et al. 2011), which would affect objects fainter than $F 814 W \sim 27$. However, the A520 images show that the slope becomes less negative for decreasing flux initially and then suddenly turns around at $\sim 400 e^{-}$. At the faintest limit, it turns around again, and the CTI is mitigatied once more.

Anderson \& Bedin (2010) developed a so-called pixelbased CTI correction method, and their standalone script PixCteCorr is publicly available and can be applied to regular FLT files. The results shown in the middle panel of Figure 1 are obtained from these images (hereafter we refer to this method as the Y2009 model) when we repeat the above experiment. The Y2009 model reduces the CTI effects substantially, and the performance is excellent especially in the brightest regime $\left(700-4000 e^{-}\right)$. Nevertheless, the model does under-correct the CTI in the intermediate flux range $\left(300-700 e^{-}\right)$. However, the most interesting feature is the behavior of the SPFs at the faintest end $\left(50-300 e^{-}\right)$. The CTI slopes are positive in this flux range because the model overcorrects the CTI. This also serves as proof of the CTI mitigation at the faint limit first reported in Jee et al. (2009) and later supported by Schrabback et al. (2010).

A new CTI correction method has been proposed by Ubeda and Anderson (2012) and it is now part of the default STScI pipeline. This method (hereafter the Y2012 model) is an important improvement over the earlier method in that it includes both time and temperature dependence. In addition, Ubeda and Anderson (2012) state that the performance in the low flux regime has been significantly improved. We display the results obtained from this new correction in the right panel of Figure 1. It is clear that the undercorrection problems (e.g., see the slope for the range $300-400 e^{-}$in the middle panel) seen in the old model nicely disappear in this case. However, unfortunately, the overcorrection problem at the faint limit still remains (perhaps becoming even slightly worse). Nonetheless the performance of this correction is the best and we use the Y2012 CTI-correction algorithm for our actual weak lensing analysis.

The remaining concern is the treatment of the over- correction problems at the faint limit. The flux range $50-300 e^{-}$corresponds roughly to $F 814 W=27-28.5$. However, this blind conversion is not accurate mainly because galaxy profiles are much less steep than those of the SPFs. Thus, any naive attempt to merely correct the ellipticity of these sources at the catalog level will likely fail. As in Jee et al. (2011), we correct for these residual CTI-correction errors by modifying our model PSF by treating the distortion of the PSF due to CTI as an additional convolution. This assumption should hold better in the current study than in Jee et al. (2011) because most significant CTI-effects are already fixed by the pixel-based method (thus, the residual CTI effect on PSF can be approximated by slightly stretching the PSF). We find that about $15 \%$ of our source galaxies need this additional correction. Because the residual correction is small and the ellipticities of these faint galaxies are already down-weighted, the impact due to the imperfection of our residual correction on weak lensing analysis is negligibly small

Another method to address the overcorrection would be simply to discard the sources at the faint end that are likely to be over-corrected by the Y2012 algorithm. We verify that our weak-lensing result obtained with this scheme is highly consistent with those from the full catalog, albeit with a slightly higher noise level.

\subsection{PSF Model}

Although the PSF of ACS is small, a careful effort must be made to model and remove the smearing effect of the PSF. In particular, when one desires to utilize sources near the $5 \sigma$ detection limit and the size of the PSF, the complex spatial variation of the ACS PSF should be fully considered to avoid bias due to the PSF anisotropy (the sizes of these faint sources are comparable to that of the PSF).

About 10-20 high S/N (> 20) stars are found in the individual exposures of the A520 data. Since the ACS PSF is both time- and position-dependent, it is impossible to use this small number of stars to derive a reliable PSF model at the location of galaxies across the field. Therefore, we utilize the PSF library of Jee et al. (2007a) 

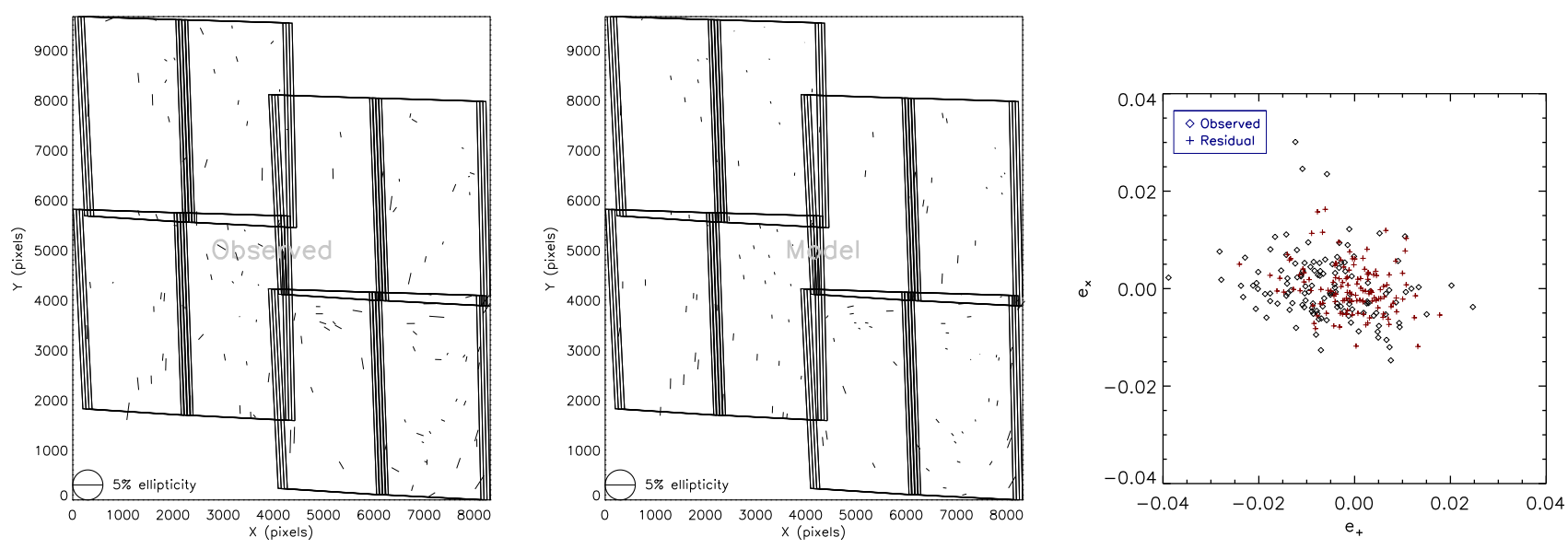

Figure 2. PSF reconstruction in the F814W images. The left panel shows the observed ellipticity pattern of the stars whereas in the middle panel we display the ellipticity of the model PSF derived from globular cluster fields. The sticks illustrate the direction and magnitude of the ellipticity of the PSFs. The solid lines represent the observation footprints. The plot in the right panel displays the residual ellipticity of the stars.

constructed from dense stellar fields. The PSF pattern of ACS is repeatable (Jee et al. 2007a), and thus can be estimated for each exposure by measuring the shape properties of the stars in the A520 data and comparing them with those from the PSF library. We keep track of the model PSFs at the locations of source galaxies in individual exposures to compute the final PSF on the stack image. This requires a rigorous propagation of the image stacking history including offset, rotation, and weight applied to individual exposures. We refer readers to our previous publication (e.g., Jee et al. 2011) for details.

Figure 2 compares the PSF ellipticity pattern of the stars and the model PSFs in the A520 F814W images, where we measure shears. The comparison shows that our PSF model reasonably mimics the observed PSF pattern. Any significant registration error is supposed to create spurious stellar ellipticities not observed in the model. We do not find any hints of such a large discrepancy. The residual PSF ellipticity rms per component is small $(\sim 0.006)$, and thus the impact of the residual anisotropy on shear is negligible.

\subsection{Shape Measurement and Cluster/Source Galaxy Separation}

Our ellipticity is defined by $(a-b) /(a+b)$, where $a$ and $b$ are the semi-major and minor axes, respectively ${ }^{10}$. We determine the ellipticity of an object by fitting a PSFconvolved elliptical Gaussian.

Because the elliptical Gaussian is not the best representation of real galaxies, it is important to correct for this "underfitting" (Bernstein 2010) together with other shear calibration issues such as the dilution of the signal by noise and spurious sources. Our internal shear calibration utilizing the Hubble Ultra Deep Field (Beckwith et al. 2006; HUDF) data shows that the average correction factor is $\sim 11 \%$. This average value is greatly influenced by low-surface brightness galaxies at $F 814 W>26$,

10 An alternative definition of shape using $\left(a^{2}-b^{2}\right) /\left(a^{2}+b^{2}\right)$ is often used in the literature. One should remember that this socalled polarisation needs to be divided by 2 to obtain an estimate of the shear. which require a slightly larger ( $\sim 14 \%$ on average) correction factor, but still contain a useful lensing signal. This $\mathrm{S} / \mathrm{N}$-dependent correction is often called noise bias (Melchior \& Voila 2012; Refregier et al. 2012), and is an important factor affecting cosmic shear results. In this paper, we multiply a $\mathrm{S} / \mathrm{N}$-independent average correction factor 1.11 to our galaxy ellipticity. However, we verify that analysis using a $\mathrm{S} / \mathrm{N}$-dependent correction scheme yields virtually indistinguishable weak-lensing results. Note that this multiplicative bias affects the amplitude of the lensing signal, but should not affect features in the mass map. To maximize the $\mathrm{S} / \mathrm{N}$ of the lensing signal, the ellipticity of galaxies must be properly weighted by taking into account both the source ellipticity distribution and measurement errors. We employ the following simple inverse-variance weighting scheme:

$$
\mu_{i}=\frac{1}{\sigma_{S N}^{2}+\left(\delta e_{i}\right)^{2}},
$$

where $\sigma_{S N}$ is the dispersion of the source ellipticity distribution ( $\sim 0.25$ per component for the A520 data), and $\delta e_{i}$ is the $i^{t h}$ galaxy's ellipticity measurement error per component.

For bright galaxies $(\mathrm{F} 814 \mathrm{~W}<24)$, we use both $\mathrm{F} 435 \mathrm{~W}$ F606W and F606W-F814W colors to select the A520 members utilizing the criteria of $\mathrm{C} 12$ (i.e., objects inside the quadrilateral in Figure 1 of C12). This gives a total of 447 objects, and we use them to estimate the cluster luminosity. For the source galaxy sample, we select galaxies between $22<F 814 W<27.5$ and remove the bright $(\mathrm{F} 814 \mathrm{~W}<24)$ cluster members. We do not attempt to remove cluster members at $\mathrm{F} 814 \mathrm{~W}>24$ because the above color-based selection is not efficient in this regime (in 3.4 we demonstrate that in fact the contamination from faint cluster members is negligible at F814W $>$ 24). Stars are identified utilizing both halflight radius and shape measurement results. Because delta-function-like features should arise for point sources after deconvolution, we require the minimum semi-minor axis to be 0.4 pixels to prevent accidental inclusion of stars. The maximum allowed ellipticity error per com- 


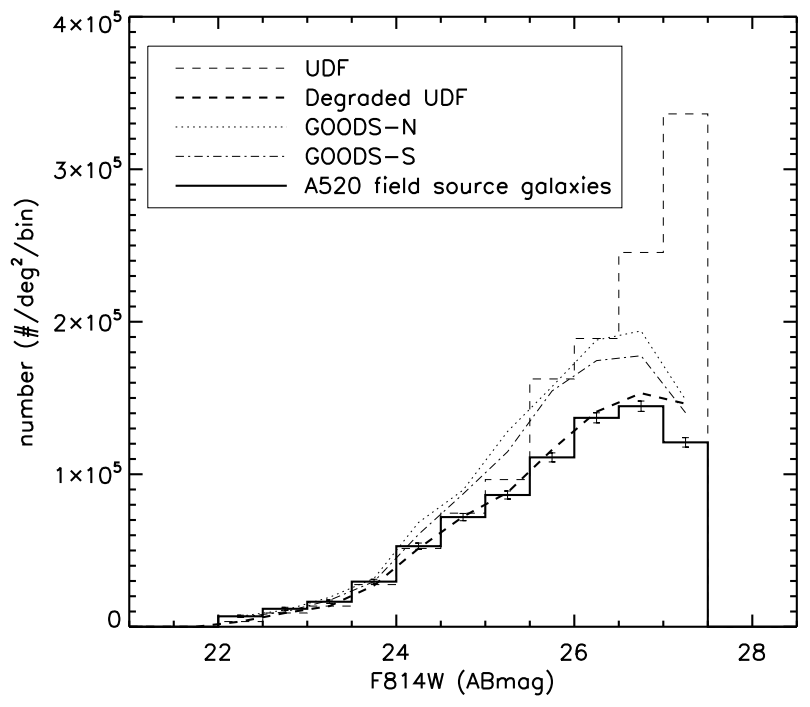

Figure 3. Magnitude distribution of source galaxies in the A520 field. We apply the identical selection (after due color transformation) criteria to the UDF and GOODS data and show the resulting magnitude distributions for comparison. For relatively bright source galaxies $(F 814 W<24.5)$, the magnitude distributions from the four fields agree nicely, indicating that the contamination from unidentified cluster members (i.e., missed by our color selection) is negligible in this regime. Because both UDF and GOODS images are much deeper than the A520 one, it is difficult to directly compare the distribution at $F 814 W \gtrsim 24.5$. Nevertheless, when we degrade these control fields in such a way that the noise levels become comparable, we observe a good agreement in this faint regime, too, up to the sample variance. The displayed error bars include only Poissonian noise.

ponent is set to 0.25 . The total number of sources after these cuts is 4,932 , giving us a source density of $\sim 109$ galaxies per sq. arcmin. C12 quotes $\sim 56$ galaxies per sq. arcmin in their ACS weak lensing analysis of A520.

According to Equation 4, the uncertainty of the shear is given by

$$
\sigma_{\gamma}=\sqrt{\frac{1}{\Sigma \mu_{i}}} .
$$

On the other hand, if no weighting scheme is used, the shear uncertainty is simply:

$$
\sigma_{\gamma}=\frac{\sigma_{S N}}{\sqrt{n}}
$$

We define the effective number by equating the last two equations and obtain

$$
n_{\mathrm{eff}}=\sum \frac{\sigma_{S N}^{2}}{\sigma_{S N}^{2}+\left(\delta e_{i}\right)^{2}} .
$$

Therefore, the effective number is always smaller than the actual number of sources. We estimate the effective source density to be $\sim 96$ per sq. arcmin. The corresponding rms shear is $\sim 0.026$ per sq. arcmin, which is $\sim 28 \%$ smaller than the value quoted by $\mathrm{C} 12(\sim 0.036$ per sq. arcmin).

\subsection{Redshift Estimation of Source Population}

Since we do not apply any color cut for galaxies fainter than $\mathrm{F} 814 \mathrm{~W} \sim 24$, it is important to estimate the level of potential contamination in our source catalog carefully and to propagate it to our redshift estimation. To address this issue, we utilize the Coe et al. (2006) HUDF photo-z catalog, the 2004 STScI release of the HUDF images (Beckwith et al. 2006), and the 2008 STScI release of the Great Observatories Origins Deep Survey (GOODS; Giavalisco et al. 2004) images. We apply the same source selection criteria to our galaxy catalog of the HUDF and GOODS data and compare their magnitude distributions with those from the source population in Figure 3 .

The comparison shows no excess of galaxies in the A520 source catalog with respect to the magnitude distribution computed from the above reference fields. At the faint end, the number densities of the galaxies in the reference fields are somewhat higher than those in the A520 field simply because of their increased depth. To enable a fair comparison, we degrade the reference images to match the noise level of the A520 images. The resulting distribution from the UDF is in good agreement with that of the A520 source galaxies. Our test with the GOODS images also confirms that the cluster galaxy contamination is negligible.

In order to scale our lensing signal properly, we must estimate the $\beta$ parameter defined as:

$$
\beta=\left\langle\max \left(0, \frac{D_{l s}}{D_{s}}\right)\right\rangle,
$$

where $D_{l s}$ and $D_{s}$ are the angular diameter distances between the lens and the source, and between the observer and the source, respectively. This $\beta$ parameter determines the critical surface mass density $\Sigma_{c}$ of the cluster given by

$$
\Sigma_{\text {crit }}=\frac{c^{2}}{4 \pi G D_{l} \beta},
$$

where $c$ is the speed of light, $G$ is the gravitational constant, and $D_{l}$ is the angular diameter distance to the lens. Compared to high-redshift clusters, the weak lensing mass of A520 at $z=0.2$ is not as sensitive to the source redshift. However, we take care to apply the same source selection criteria to the UDF data.

Using only the color and magnitude criteria gives $\beta=$ 0.75, which corresponds to an effective source plane at $z_{\text {eff }} \sim 1$. This value is slightly biased high because there are fewer galaxies at the faint limit in the A520 data than in the UDF image. In addition, the ellipticities of these faint galaxies are down-weighted when we estimate shear. Considering both effects, the revised estimate becomes $\beta=0.73$ or $z_{\text {eff }} \sim 0.85$. The resulting critical mass density $\Sigma_{\text {crit }}$ is $3.35 \times 10^{3} M_{\odot} \mathrm{pc}^{-2}$.

C12 quote $\Sigma_{\text {crit }}=3.6 \times 10^{3} M_{\odot} \mathrm{pc}^{-2}$ for their weak lensing analysis. This higher surface mass density implies that their source redshift is lower than ours, which is consistent with the fact that our source catalog contains more faint galaxies than theirs 11 .

11 We note that C12 estimate the critical surface mass density of $\mathrm{J} 12$ to be $\Sigma_{\text {crit }} \simeq 4.1 \times 10^{3} M_{\odot} \mathrm{pc}^{-2}$ using $\beta=0.64$ quoted in J12. However, substituting this $\beta$ value into equation 9 yields $\Sigma_{\text {crit }} \simeq 3.8 \times 10^{3} M_{\odot} \mathrm{pc}^{-2}$; the difference in the assumed cosmological parameters between J12 and C12 makes only a negligible change in the conversion of $\beta$ to $\Sigma_{\text {crit }}$. 


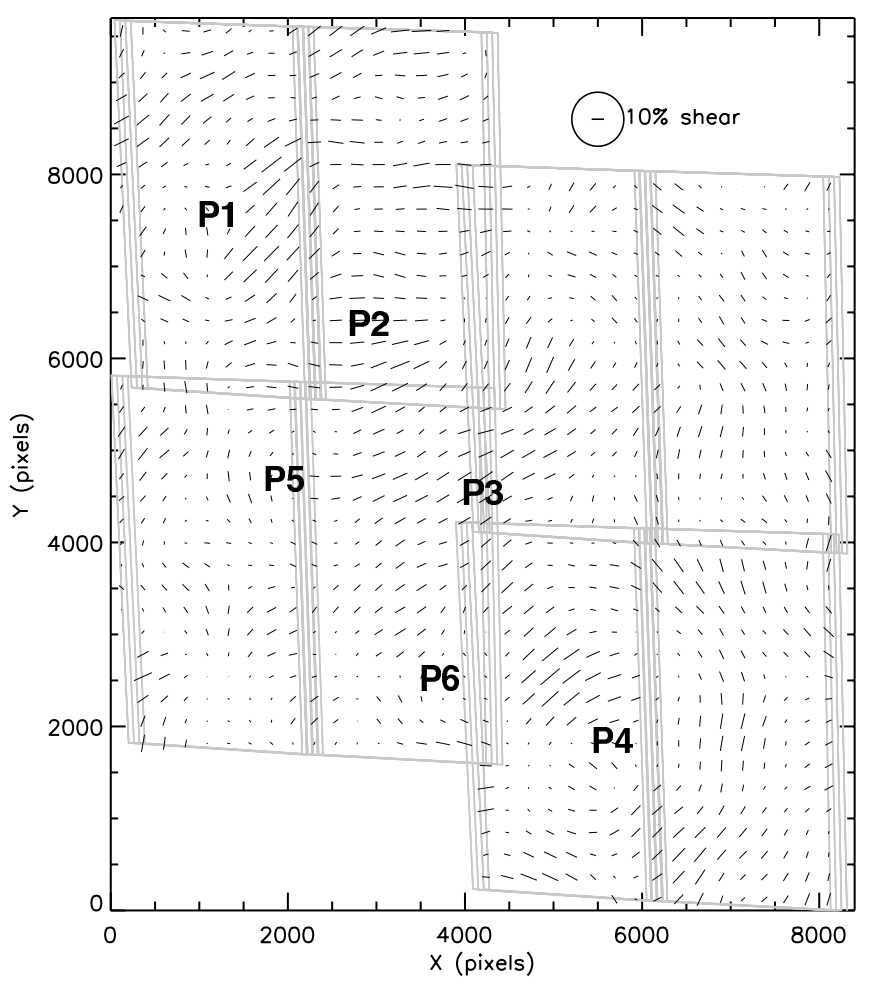

Figure 4. Smoothed ellipticity distribution of source galaxies. The "whisker" plot is produced by convolving the ellipticities with a Gaussian kernel. The diameter of the circle represents the FWHM $\left(30^{\prime \prime}\right)$ of the convolution kernel while the stick inside this circle shows a $10 \%$ horizontal shear. Clear correlation of source galaxy ellipticity is seen. The approximate locations of the substructures reported in J12 are annotated with P1-P6.

\section{MASS RECONSTRUCTION}

An important application of weak gravitational lensing is that the observed shear signal can be used to reconstruct the projected mass density. The smoothed shear field presented in Figure 4 shows a coherent pattern around the main galaxy overdensities. This can be related directly to the convergence map $\kappa(\mathbf{x})=\Sigma(\mathbf{x}) / \Sigma_{\text {crit }}$ through

$$
\kappa(\mathbf{x})=\frac{1}{\pi} \int D^{*}\left(\mathbf{x}-\mathbf{x}^{\prime}\right) \gamma\left(\mathbf{x}^{\prime}\right) d^{2} \mathbf{x} .
$$

where $D^{*}(\mathbf{x})$ is the complex conjugate of the convolution kernel $D(\mathbf{x})=-1 /\left(x_{1}-\mathbf{i} x_{2}\right)^{2}$ and $\gamma(\mathbf{x})$ is the complex representation of gravitational shear.

A better result is produced if we do not pre-smooth the ellipticities, but instead let the strength of the shear signal determine the local smoothing scale. One such method is the maximum-entropy-regularized mass reconstruction first introduced by Seitz et al. (1998). In this study, we use the Jee et al. (2007b) implementation of the method. Figure 5 shows the result. A similar result is obtained when we use equation 10, although the map becomes noisier near the edges.

The substructures seen in this ACS analysis are in general similar to those in the WFPC2 results of J12 and their locations are denoted as P1-P6; their coordinates are listed in Table 1. However, one of the important differences is the location of the dark peak. The current centroid $\left(\mathrm{P} 3^{\prime}\right)$ is about $1^{\prime}$ shifted to the southwest com- pared to the one in J12 (P3). Although mass reconstructions using different imaging data can sometimes result in small offsets in the positions of the mass peaks, it is unusual to observe a shift as large as $\sim 1^{\prime}$.

Another noteworthy difference between our ACS and WFPC2 results is the strength of the substructure $\mathrm{P} 4$. In our ACS result, $\mathrm{P} 4$ is the strongest mass peak in the A520 field whereas it appears as a minor (much weaker than P3) clump in our WFPC2 analysis. However, this difference can arise from the incomplete coverage of the region by the WFPC2 observation. In addition, we find that the ACS images reveal many new arclets around $\mathrm{P} 4$, which all contribute to the significance of the peak in mass reconstruction. We note that the inferred projected mass of P4 is consistent with the value reported in J12.

\subsection{Centroid and Significance of the Dark Peak in A520}

We perform a bootstrapping analysis to measure both the significance and the positional uncertainty of the substructures. Utilizing the fast Fischer \& Tyson (1997) implementation FIATMAP of the KS93 algorithm, we generate 1000 realizations. In Figure 6, we display nine random samples of the bootstrapped mass reconstructions. The circle denotes the approximate location of the dark peak

We measure the centroids using the first moments weighted by a circular Gaussian, whose FWHM matches the size of the substructure. The results are displayed in Table 1. The mean positional uncertainty is small $\left(\sim 5^{\prime \prime}\right)$, and thus we conclude that the large shift of the dark peak centroid between the current study and J12 is not caused by noise in the mass reconstruction. We discuss a number of possible explanations in 4.4. Nevertheless, we note that the aperture mass within $r=150 \mathrm{kpc}$ centered on P3 (old centroid) is still consistent with the WFPC2 value (see 95). In other words, there is significant dark mass present in projection about P3.

Quantifying the significance of the P3' substructure requires us to determine a reasonable baseline in the absence of the dark peak. We make a conservative estimate of this baseline by (1) taking the luminosities of all the galaxies in the $\mathrm{P} 3^{\prime}$ region (see $\$ 5.1$ for details), (2) calculating the dark matter mass assuming a fiducial $M / L$ of $300 M_{\odot} / L_{B \odot}$ (higher than the M07 value of 232, and so more conservative), and (3) adding to this dark matter mass the maximum gas mass along the column. For the X-ray gas mass, we adopt the upper limit $M_{\text {gas }}=0.85 \times 10^{13} M_{\odot}$. The resulting mean convergence (adding both dark matter and gas masses) within the $r<150 \mathrm{kpc}$ aperture is $\sim 0.04$. Because the FIATMAP convergence maps are subject to mass-sheet degeneracy, we rescale the maps in such a way that the substructure masses agree with those derived from aperture mass statistics. The significance is computed by first subtracting the baseline value from the rescaled convergence within the $r=150 \mathrm{kpc}$ aperture and then dividing the result by the rms obtained from the 1000 runs. The distribution in the significance of the $\mathrm{P} 3^{\prime}$ is shown in Figure 7 . The mean of the distribution is $\sim 6.6 \sigma$, and the low-end tail is $>4 \sigma$. This mean value $\sim 6.6 \sigma$ is similar to the significance estimate based on our aperture mass densitometry (\$5.1). Also displayed in Figure 7 is the significance distribution when we repeat the experiment 

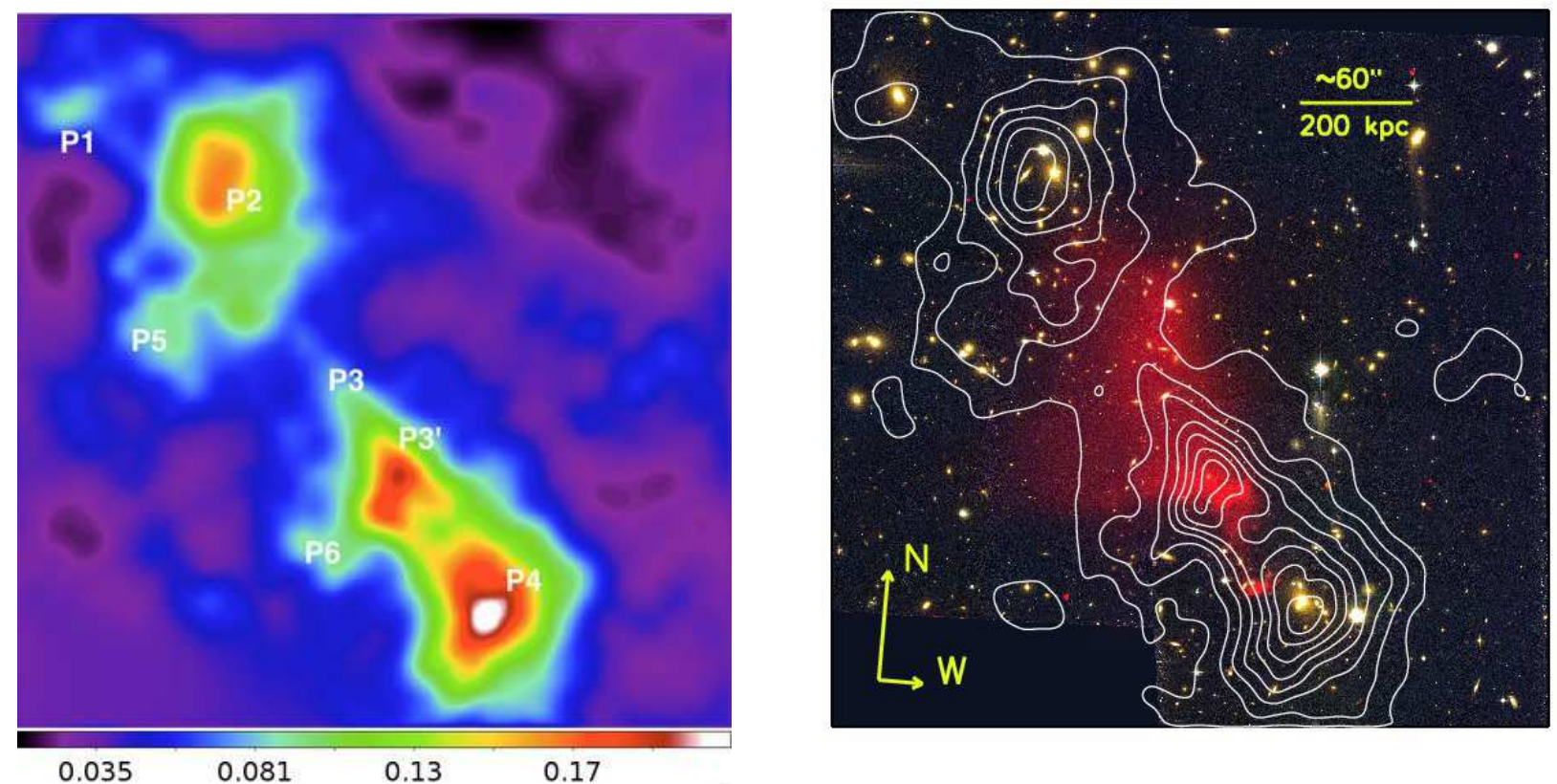

Figure 5. Maximum-entropy-regularized mass reconstruction of A520. The left panel shows the density distribution using the color scheme shown at the bottom. We annotate the location of the substructures reported in J12 as P1-P6. On the right panel, we overlay the mass contours on the ACS color composite image. The intensity of the X-ray emission observed by Chandra is represented in red.

Table 1

Mass Properties of Substructure $(r<150 \mathrm{kpc})$

\begin{tabular}{|c|c|c|c|c|}
\hline Substructure & $\begin{array}{c}\alpha, \delta \\
\left(h \begin{array}{cc} & s, \circ,^{\prime \prime}\end{array}\right)\end{array}$ & $\begin{array}{c}\Delta \alpha, \Delta \delta \\
\left({ }^{\prime \prime},{ }^{\prime \prime}\right)\end{array}$ & $\begin{array}{c}\text { Projected Mass } \\
\left(h_{70}^{-1} 10^{13} M_{\odot}\right)\end{array}$ & $\begin{array}{c}\text { Gas Mass } \\
\left(h_{70}^{-5 / 2} 10^{13} M_{\odot}\right)\end{array}$ \\
\hline $\begin{array}{l}\text { P1 } \\
\text { P2 } \\
\text { P3 (old centroid) } \\
\text { P3' (new centroid) } \\
\text { P4 } \\
\text { P5 }\end{array}$ & 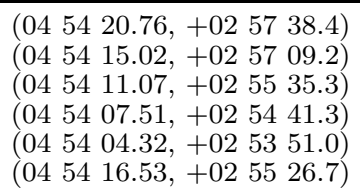 & $\begin{array}{c}(5.3,4.1) \\
(4.0,4.9) \\
- \\
(5.1,6.2) \\
(3.0,4.4) \\
(5.4,8.2)\end{array}$ & $\begin{array}{l}2.10 \pm 0.43 \\
4.05 \pm 0.28 \\
3.35 \pm 0.34 \\
3.94 \pm 0.30 \\
4.23 \pm 0.28 \\
2.93 \pm 0.39\end{array}$ & $\begin{array}{l}<0.23 \\
<0.40 \\
<0.74 \\
<0.85 \\
<0.34 \\
<0.21\end{array}$ \\
\hline
\end{tabular}

Note. - The positional uncertainty is estimated from bootstrapping. The mass uncertainties are evaluated from 1000 Monte-Carlo realizations. The gas mass is derived using Cauchy-Schwartz method in M07 based on the data set ObsID 9426 (110 ks) for P3' and ObsId 528 (38 ks) for the other peaks. The Cauchy-Schwarz method is a model-independent means of deriving an upper limit on the gas mass column in a given region of the sky. The method requires an estimate of the maximum length of the cluster gas along the line of sight that contributes $99 \%$ of the emission. We refer to M07 for details; here we use an updated maximum column length of $4 \mathrm{Mpc}$ (instead of 2), which is a more conservative estimate given the total mass of Abell 520. As a result, our gas mass upper limits are more conservative, being higher than M07 by $\sqrt{2}$. The given values are $90 \%$ confidence level upper limits.

with the C12 weak-lensing catalog. The dark peak is still present in $\mathrm{C} 12$, although the significance in C12 is systematically lower. The reason is two-fold. C12 used a factor of two fewer source galaxies, and the convergence in the P3' slightly lower. This catalog-based comparison is detailed in 6 .

\subsection{Impact of the CTI correction model}

As discussed in $\S 2$, CTI is an important instrumental effect and although the Y2012 method (Ubeda \& Anderson 2012) that we use in our analysis is not perfect, our tests on SPFs presented in Figure 1 shows it performs better than the Y2009 correction (Anderson \& Bedin 2010) down to fluxes of $300 e^{-}$. One common misconception regarding the CTI effect on weak lensing mass reconstruction is that it affects only the region where the readout distance is the largest. However, this is not an accurate statement especially in the case of two-dimensional mass reconstructions, which are related to shear fields non-locally. It is therefore interesting to examine whether or not the residual CTI features affect our weak lensing analysis.

In Figure 8 we compare the Kaiser \& Squires (1993; hereafter KS93) mass reconstruction results when we do not correct for CTI (left panel), use the Y2009 method (middle panel) or the most recent approach (Y2012; right panel). Note that C12 used the Y2009 method as well as an updated model from Massey et al. (2010) and claimed consistent results in both cases. We assume the equality $g=\gamma$ because an iterative nonlinear reconstruction using the relation $g=\gamma /(1-\kappa)$ may exaggerate the difference 


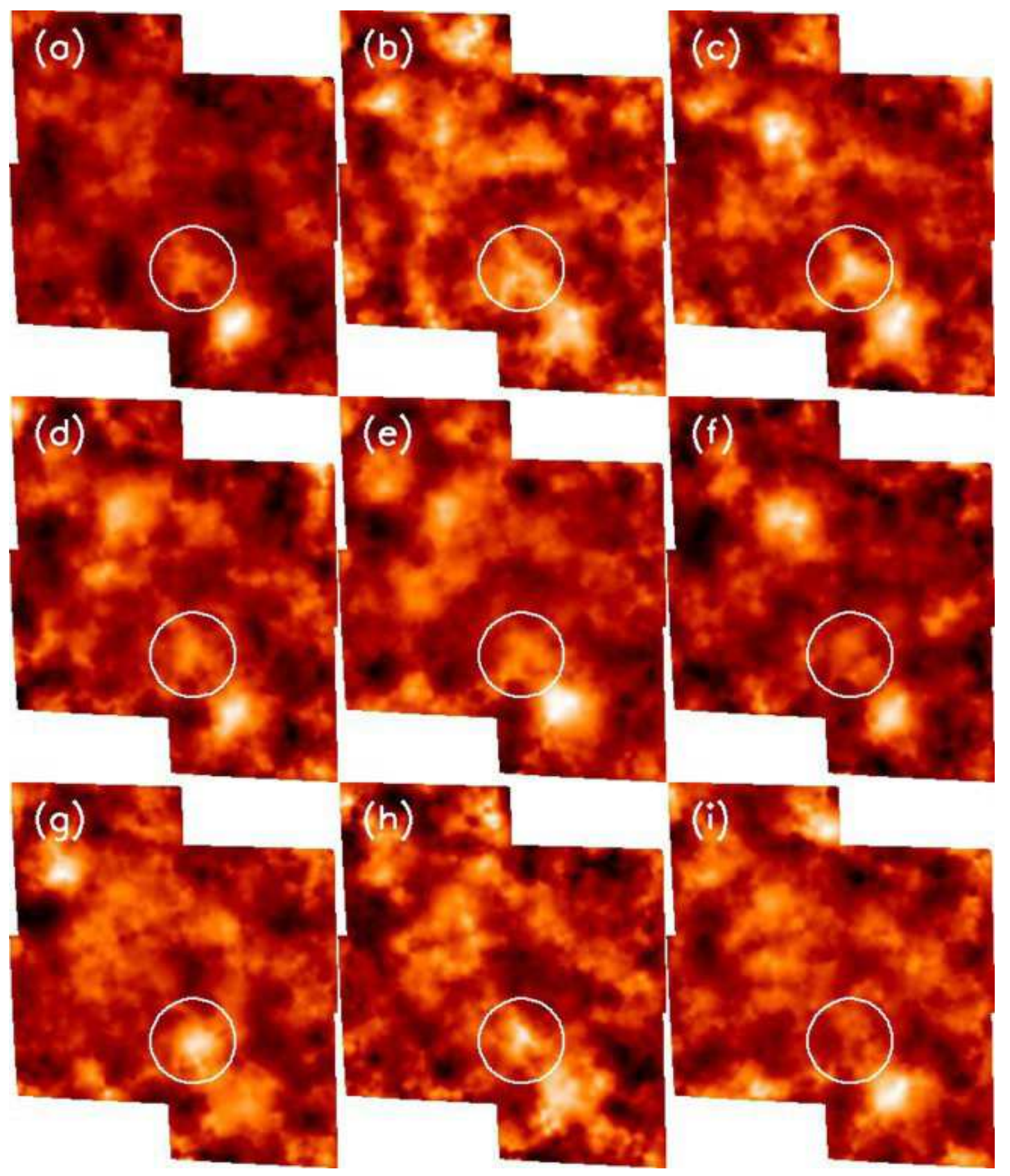

Figure 6. Bootstrapping test of the A520 mass reconstruction. Mass reconstruction is performed with bootstrap-resampled source galaxies. The test provides a measure to examine the statistical significance of the A520 substructures. We use the FIATMAP code to generate 1000 results. Here we display nine random results. The circle denotes the approximate location of the dark peak.

among the different versions.

The overall distributions of the three convergence fields are similar to one another without any conspicuous substructure only seen in any particular version. However, it is important to note that the relative strengths of the mass peaks are significantly different. The most critical substructure in A520 is the overdensity P3' (indicated by the white arrow) between the two dominant mass peaks, where there are no luminous cluster members. It is remarkable that the overdensity in this region is strongest when we apply the best-performing Y2012 CTI correction method (right panel). The feature is still seen, but weakest when no CTI correction is applied (left panel). The Y2009 correction gives an intermediate significance.
As shown in Figure 8, the assessment of the substructure significance relative to another is influenced by imperfect CTI correction. Therefore, we conclude that the impact of the fidelity of the CTI correction is nonnegligible in weak lensing analysis with the current A520 data. We revisit this issue when we discuss our actual mass determinations in $\$ 5$.

\subsection{Comparison with the WFPC2 analysis}

The most noteworthy difference in the weak lensing results between our current ACS and the WFPC2 study is the large shift of the centroid of the dark peak from $\mathrm{P} 3$ to $\mathrm{P} 3^{\prime}$ by $\sim 1^{\prime}$. Since this shift is much larger than the centroid uncertainty determined from the bootstrapping 


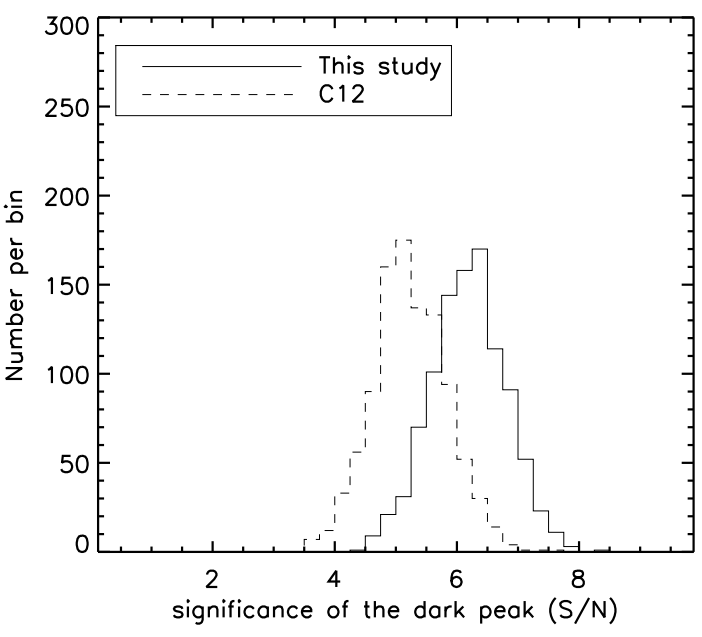

Figure 7. Significance of the dark peak measured from our 1000 bootstrap runs. The signficance is measured using a $r=150 \mathrm{kpc}$ aperture against the background, which is determined within each mass reconstruction field. See text for details.

experiment (Table 1), it is difficult to attribute the shift to mere shot noise. Here we examine whether the shift originates from a systematic difference between the two source catalogs of ACS and WFPC2.

We first carry out a galaxy-by-galaxy comparison of the two catalogs. Figure 9 shows that the raw ellipticities (prior to the application of shear calibration) between ACS and WFPC2 agree nicely. The average slope of the two ellipticity components is $\sim 1.05$. This small departure from unity disappears when we apply the appropriate shear calibration factor to each dataset. Hence this comparison does not indicate a systematic difference in ellipticity.

To examine whether any systematic shape error might be localized near the dark peak we perform a weak lensing mass reconstruction using the shapes of the ACS data but based on the source selection in J12. In our WFPC2 catalog there are more (less) source galaxies in the P3 $\left(\mathrm{P} 3^{\prime}\right)$ region than in our ACS catalog. Thus, the number density distribution of the common source galaxies does not exactly match the source density distribution of the WFPC2 data. We return to the issue of source density below.

Figure 10] shows the convergence field obtained from this source catalog using the KS93 method. Interestingly, the location of the dark core now coincides with that of P3 in J12 in this mass reconstruction. This demonstrates that the shape catalogs based on either WFPC2 or ACS data give consistent results when a similar source selection is made.

\subsection{Origin of the shift in peak location}

While matching objects between the WFPC2 and ACS catalogs, we found that there is a systematic difference in source galaxy density distribution. Figure 11$]$ compares the source galaxy distribution between the ACS and WFPC2 data. It is clear that in the WFPC2 cata$\log$ the density in the $\mathrm{P} 3$ region is much higher than the surrounding area. This is mainly because the WFPC2 observation was planned in such a way that much deeper imaging is performed in this region (see also Figure 2 of J12). Although we still hold to the claim of J12 that the spatial variation of the source galaxy number density does not cause any spurious substructure, it appears that the centroid of the dark peak is influenced by this large inhomogeneity of the source galaxy distribution.

We suspect that the elongation of the substructure in the dark peak region along the merger axis also facilitates the centroid shift. This particular shape of the substructure should make its centroid more uncertain along the P3-P3' orientation and sensitive to the source density fluctuation near the dark peak region. The CFHT location of the dark peak in M07 is similar to that of J12, although it is closer to P3' by $\sim 20^{\prime \prime}$ than in J12. However, given the large smoothing scale (because of the relatively small number of source galaxies) of the CFHT mass reconstruction, we do not consider the centroid difference between M07 and the current study very significant (see the centroid variation in Figure 7 of M07 for different images).

Finally, we discuss the effect of cluster galaxy contamination. In J12, the cluster member selection is based on the CFHT $g-r$ color, mainly identifying the redsequence galaxies of A520 except for some blue galaxies whose spectroscopic redshifts are known. The current ACS selection based on three filters approximately increases the number of the cluster member candidates by $\sim 60 \%$ (295 vs. 474 candidates). Most of this increase comes from increasing the number of relatively faint blue cluster member candidates whose F435W-F606W colors are less than $\sim 1.3$. However, this improvement in the cluster member removal has a negligible impact on the weak lensing analysis for the following reasons. First, the blue cluster member selection is still unreliable even when one uses three broadband photometry. Second, we find that the spatial distribution of these additional blue cluster candidates does not correlate with the substructures. Third, most importantly, our mass reconstruction using the source galaxy catalog where we only remove the red-sequence is very similar to the results presented in Figure 5 .

\section{MASS ESTIMATES}

It is possible to use the two-dimensional mass map to measure substructure masses, but the results are sensitive to the algorithm used. More importantly, on small scales the result depends on details such as smoothing scheme, treatment of non-linearity, etc. Also, one can consider fitting halo models (e.g., NFW) to all mass clumps simultaneously. This is feasible when the substructures are simple and sufficiently massive such as those of the "El Gordo" cluster (Jee et al. 2013). We find that this simultaneous fit becomes unstable when applied to A520, which consists of at least five mass clumps within the $r \sim 0.5 \mathrm{Mpc}$ region.

Therefore, we report our mass estimates based on the aperture mass densitometry (Fahlman et al. 1994; Clowe et al. 2000), which provide a more direct estimate of the projected mass by measuring the tangential shear as a function of radius around the position of interest.

The aperture mass densitometry also allows us to take full advantage of the large field of view provided by our ancillary Canada France Hawaii Telescope (CFHT) data, which is important for efficiently breaking the so-called 


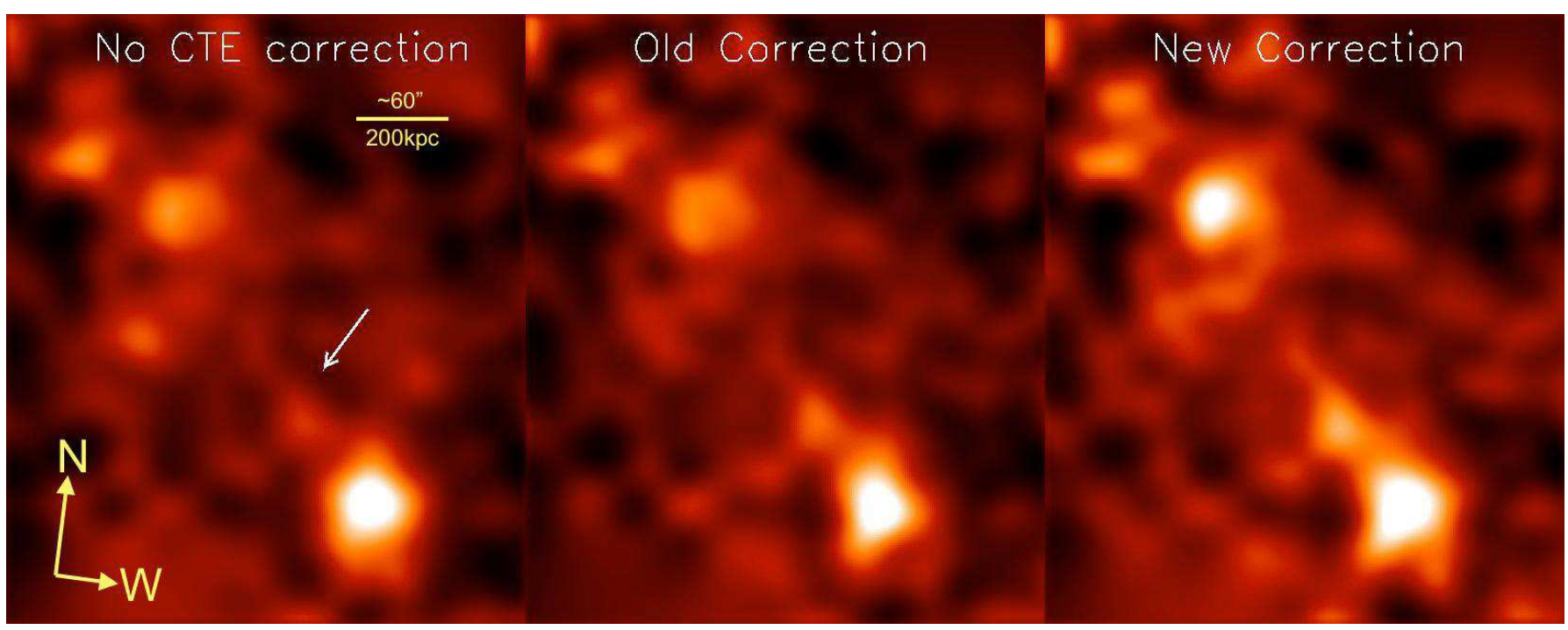

Figure 8. Influence of CTI on the A520 mass reconstruction. We present mass reconstruction results based on the KS93 method for different CTI-correction schemes. The left, middle, and right panels correspond to the cases of the left, middle, and right panels in Figure 1 respectively. Although the CTI effect alone does not introduce any artificial substructures. Incomplete corrections will cause inaccurate representation of the relative strengths among different substructures. The arrow indicates the location which we identify as a new centroid of the dark core. It is clear that the significance of this substructure will be underestimated if the CTI correction is less than optimal. We use the same intensity-to-color mapping scheme for the representation of the three mass maps after normalizing the result by the maximum convergence value of each map.
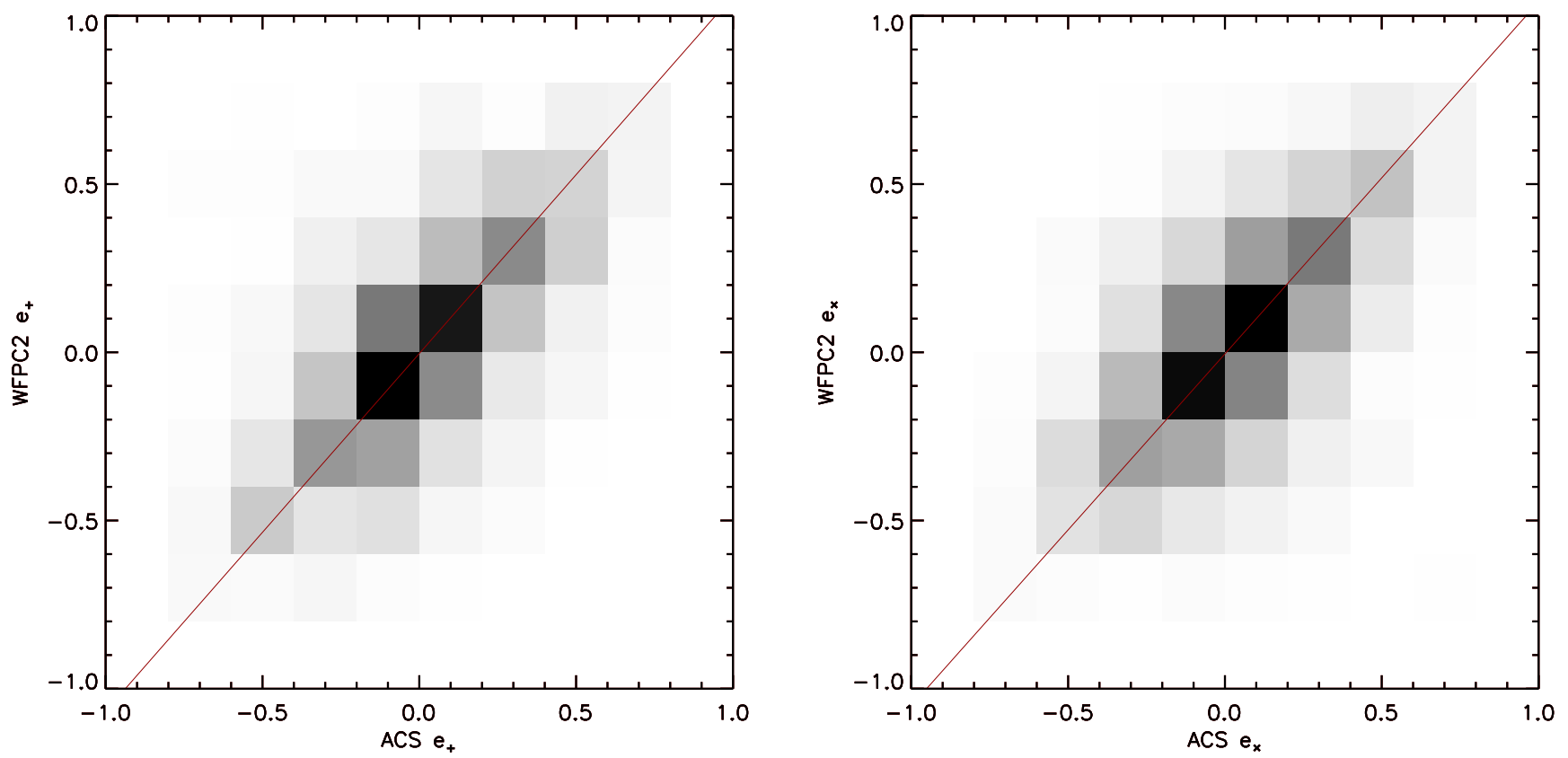

Figure 9. Ellipticity comparison between ACS and WFPC2. We compare the raw ellipticities that are directly obtained from elliptical Gaussian fitting. A total of 1764 source galaxies are common to both shape catalogs. PSF and CTI effects are corrected, but no shear calibration is applied. The J12 ellipticities from the WFPC2 image agree nicely with those from the current ACS data. The average slope of the two panels is $\sim 1.05$. This small departure from unity disappears when we apply the due shear calibration factor to each dataset. 


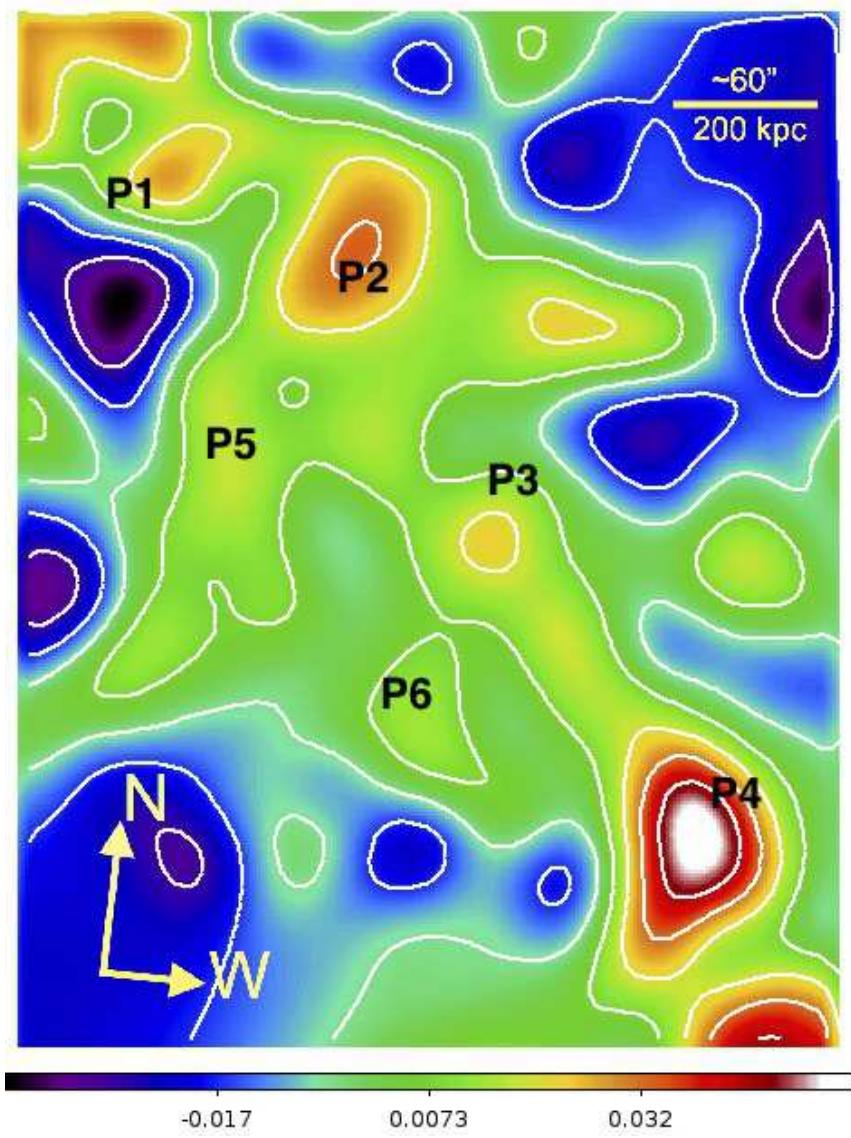

Figure 10. Mass reconstruction test using the source selection in J12. The galaxy shapes are measured from the current ACS data. We use the KS93 method to compute the convergence field by assuming $g=\gamma$. Interpretation requires caution because the field boundary defined by the overlapping region between the ACS and WFPC2 images is complicated. Nevertheless, we confirm that in this mass reconstruction the location of the dark core agrees with that in J12.

mass-sheet degeneracy. C12 combined ACS and Magellan data in their aperture densitometry to overcome the small field of view of ACS. Although both this study and C12 supplement the ACS data with ground-based images, we believe that the main differences arise from the different treatments of the ACS data.

As is done in J12, we combine the shape catalogs from the CFHT and ACS images by preferring ACS shapes wherever available. The difference in the effective source plane redshift between ACS and CFHT is accounted for by scaling up the CFHT shears by the amount required for the difference in the redshift. We verify that the amount of correction due the mean source redshift difference is consistent with the difference in the amplitude of the raw tangential shear profiles in the overlapping region.

The tangential shear is defined as

$$
g_{T}(r)=-g_{+}(r) \cos 2 \phi-g_{\times}(r) \sin 2 \phi,
$$

where $\phi$ is the position angle of the object with respect to the reference point. We use the symbol " $g$ " to remind readers that the measured quantities are in fact reduced shears.

In Figure 12, we present the tangential shear profiles derived from this combined shape catalog around P3 (left) and P3' (right). The open circles represent the results from our "null" (45-deg rotation) test and show that this so-called B-mode signal is statistically consistent with zero. The best-fit isothermal profiles using the data at $r>200^{\prime \prime}$ (dashed) predict a velocity dispersion of $1077 \pm 44 \mathrm{~km} / \mathrm{s}$. The best-fit NFW profile using the Duffy et al. (2008) mass-concentration relation gives $M_{200}=9.6_{-1.2}^{+1.4} \times 10^{14} M_{\odot}\left(r_{200}=1.91 \pm 0.09\right.$ $\mathrm{Mpc} ; c=3.21 \pm 0.04 \sqrt{12}$. Although we quote these values based on the tangential shears around P3, little difference is observed as to the total mass of the cluster when we select $\mathrm{P}^{\prime}$ as a reference point. C12 report $M_{200}=(9.1 \pm 1.9) \times 10^{14} M_{\odot}$ by assuming $c=3.5$. Their $M_{200}$ mass is consistent with ours.

A good agreement in terms of the global mass is also seen when we compare projected masses. C12 estimate that within $r=700 \mathrm{kpc}$ the aperture mass is $(5.1 \pm 0.7) \times$ $10^{14} M_{\odot}$, which is close to our estimate $(5.72 \pm 0.36) \times$ $10^{14} M_{\odot}$.

The aperture mass statistics can be evaluated by performing the following integral:

$$
\begin{aligned}
\zeta_{c}\left(r_{1}, r_{2}, r_{\max }\right) & =\bar{\kappa}\left(r \leq r_{1}\right)-\bar{\kappa}\left(r_{2}<r \leq r_{\max }\right) \\
& =2 \int_{r_{1}}^{r_{2}} \frac{\left\langle\gamma_{T}\right\rangle}{r} d r+\frac{2}{1-r_{2}^{2} / r_{\max }^{2}} \int_{r_{2}}^{r_{\max }} \frac{\left\langle\gamma_{T}\right\rangle_{r}}{r}(12)
\end{aligned}
$$

where $\left\langle\gamma_{T}\right\rangle$ is the azimuthally averaged tangential shear, $r_{1}$ is the aperture radius, and $r_{2}$ and $r_{\max }$ are the innerand the outer radii of the annulus. It is important to iteratively update $\gamma$ using $\gamma=(1-\kappa) g$ where $\kappa$ is nonnegligible. Because $\zeta_{c}\left(r_{1}, r_{2}, r_{\max }\right)$ provides a density contrast of the region inside $r<r_{1}$ with respect to the control annulus $\left(r_{2}, r_{\max }\right)$, one desires to choose $r_{2}$ and $r_{\max }$ to be large so that the mean density in the control annulus becomes small and mostly limited by the large scale structure (i.e., cosmic shear) of the field. In this paper, we choose the annulus defined by $r_{2}=600^{\prime \prime}$ and $r_{\max }=800^{\prime \prime}$. When P3 is selected as the reference, the mean density of this region is estimated to be $\bar{\kappa}=0.018$ $(\bar{\kappa}=0.009)$ according to the SIS $(\mathrm{NFW})$ profile fitting result (Figure 12). This factor of two difference in the density estimates of the annulus causes only a $\sim 5 \%$ difference in the substructure masses within the $r=150 \mathrm{kpc}$ radius. In this paper, we adopt the SIS fitting values for consistency with J12.

As for error propagation in aperture mass densitometry, we perform 1000 Monte Carlo simulations by randomizing the tangential shear profiles. Neither the difference in the background density estimation nor the effect of the cosmic shear (Hoekstra 2001, 2003) is included in our error propagation. We note that the latter is not important on small scales.

Table 1 lists the substructure masses obtained from the current aperture mass densitometry. We leave out the substructure P6 because its $r=150 \mathrm{kpc}$ circle substantially overlaps with that for $\mathrm{P} 3^{\prime}$. We do list the results for P3 to enable a comparison with the results from J12 and $\mathrm{C} 12$.

The aperture mass centered on $\mathrm{P} 3^{\prime}$ is $(3.94 \pm 0.30) \times$ $10^{13} M_{\odot}$, whereas for P3 we find $(3.35 \pm 0.34) \times 10^{13} M_{\odot}$.

12 By $M_{200}$, we define the total within the radius for which the mean internal density is 200 times the critical density. 


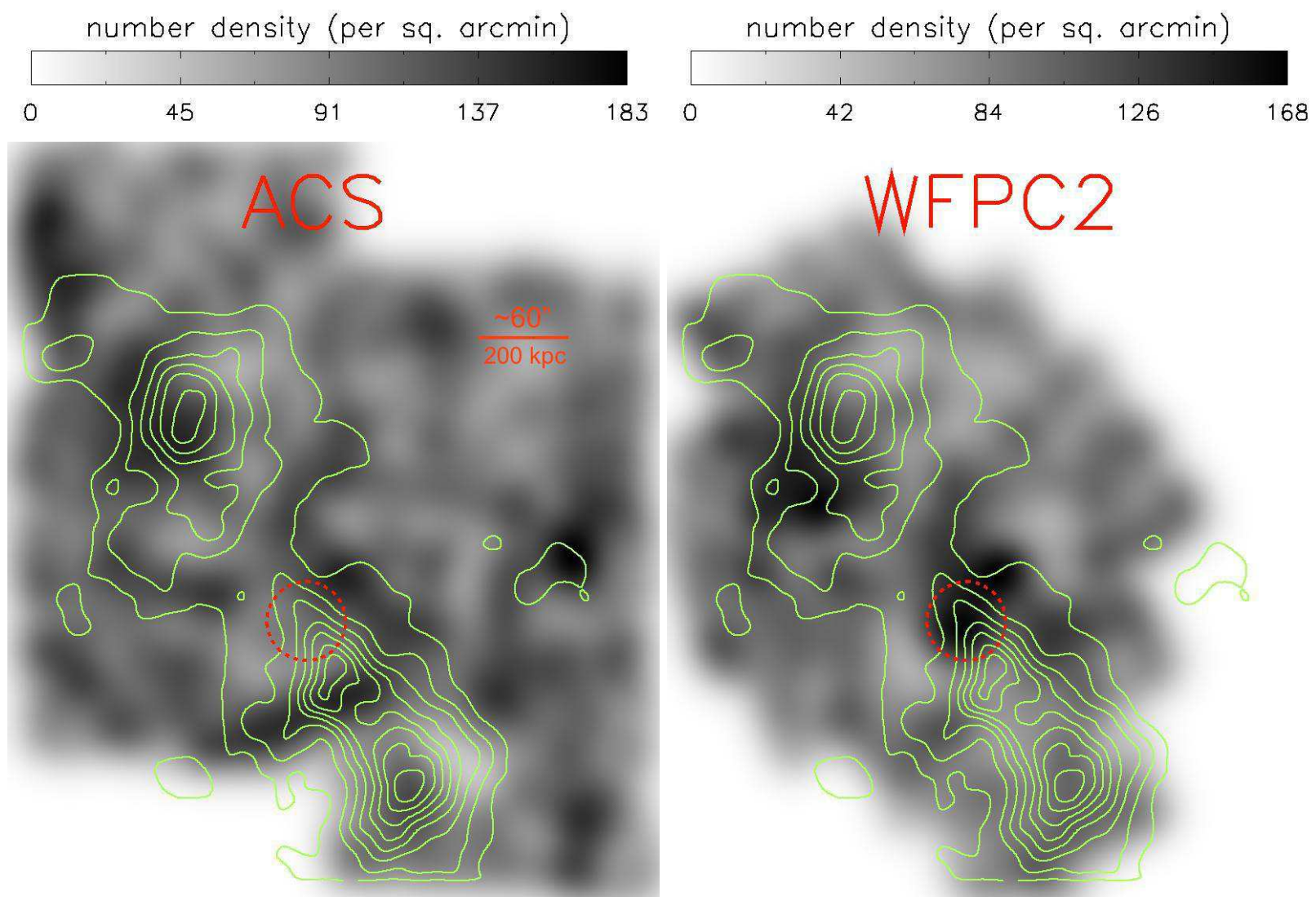

Figure 11. Difference in the source galaxy distribution between ACS (left) and WFPC2 (right) data. We smooth the source galaxy distribution using a Gaussian with FWHM $=30^{\prime \prime}$. Overlaid are the convergence contours derived from the ACS data. We argue that the difference in the source density fluctuation might have caused the centroid shift of the substructure P3 in J12. One of the largest difference between the two source density maps is seen near P3 (red circle), where the WFPC2 shows a strong concentration relative to the neighboring region whereas this contrast is not clear in the ACS data.

The latter value is lower than that of $\mathrm{J} 12$ by $\sim 16 \%$, but the error bars of the two results overlap. C12 quotes an even lower value $(2.84 \pm 0.64) \times 10^{13} M_{\odot}$ from their aperture mass densitometry. This estimate is statistically consistent with our current result, mainly because of their larger errors. When we use the ACS shape catalog matched to the WFPC2 observations, we obtain $(3.66 \pm 0.35) \times 10^{13} M_{\odot}$, in better agreement with the J12 value.

As both this study and C12 analyze the same ACS data, perhaps this $16 \%$ discrepancy in the central value is an indicator of a systematic difference between the two studies. The C12 mass of P3 is in slight tension with the J12 and M07 values at the $\sim 2 \sigma$ level. A similar level of variations exists for other substructures as well. For example, the current aperture mass of $\mathrm{P} 4$ is $(4.23 \pm$ $0.30) \times 10^{13} M_{\odot}$, whose central value is about $16 \%$ higher than the J12 result, although again the two results are statistically consistent. C12 estimated the mass of P4 to be $5.59 \pm 0.68$. This result is $\sim 32 \%$ higher than the result estimated in this study, and the discrepancy is larger than in the case of the P3 comparison.

The ratio of the aperture mass of $\mathrm{P} 3^{\prime}$ to that of $\mathrm{P} 3$ in our ACS analysis is $\sim 1.17$, which may appear discordant with the visual impression of a larger difference that one receives from the mass reconstruction (Figure 5). We attribute this difference to the different range of tangential shears affecting each result: the aperture mass densitometry uses only the information outside the aperture radius $\left(r>r_{1}\right)$ whereas the mass reconstruction is influenced by the shear signal inside the aperture, as well as the information outside the aperture. Therefore, the factor of two higher amplitude of the tangential shear (Figure 12) at the inner most bin, which is not included in the aperture mass statistics, is responsible for the larger contrast between $\mathrm{P} 3$ and $\mathrm{P} 3^{\prime}$ in our ACS mass reconstruction.

In 3.1 we demonstrate that the fidelity of the CTI correction non-negligibly affects the mass reconstruction results. The substructure around the dark peak region becomes strongest when the latest Y2012 model (Ubeda and Anderson 2012) is applied. The feature becomes weaker when the Y2009 CTI correction method (Anderson \& Bedin 2010) is used, and it becomes weakest when we do not apply any CTI correction. We observe a consistent trend in aperture mass densitometry. With the Y2009 CTI correction, we obtain $M_{a p}(r<$ $150 \mathrm{kpc})=(3.09 \pm 0.33) \times 10^{13} M_{\odot}$ for P3, 8\% lower than the above $(3.35 \pm 0.34) \times 10^{13} M_{\odot}$. When no CTI correction is applied, the resulting aperture mass becomes $M_{a p}(r<150 \mathrm{kpc})=(2.87 \pm 0.43) \times 10^{13} M_{\odot}, \sim 14 \%$ lower than the result that we obtain with the latest CTI model. On the other hand, we find that the aperture mass of P4 increases by $\sim 10 \%$, when no CTI correction is applied. 

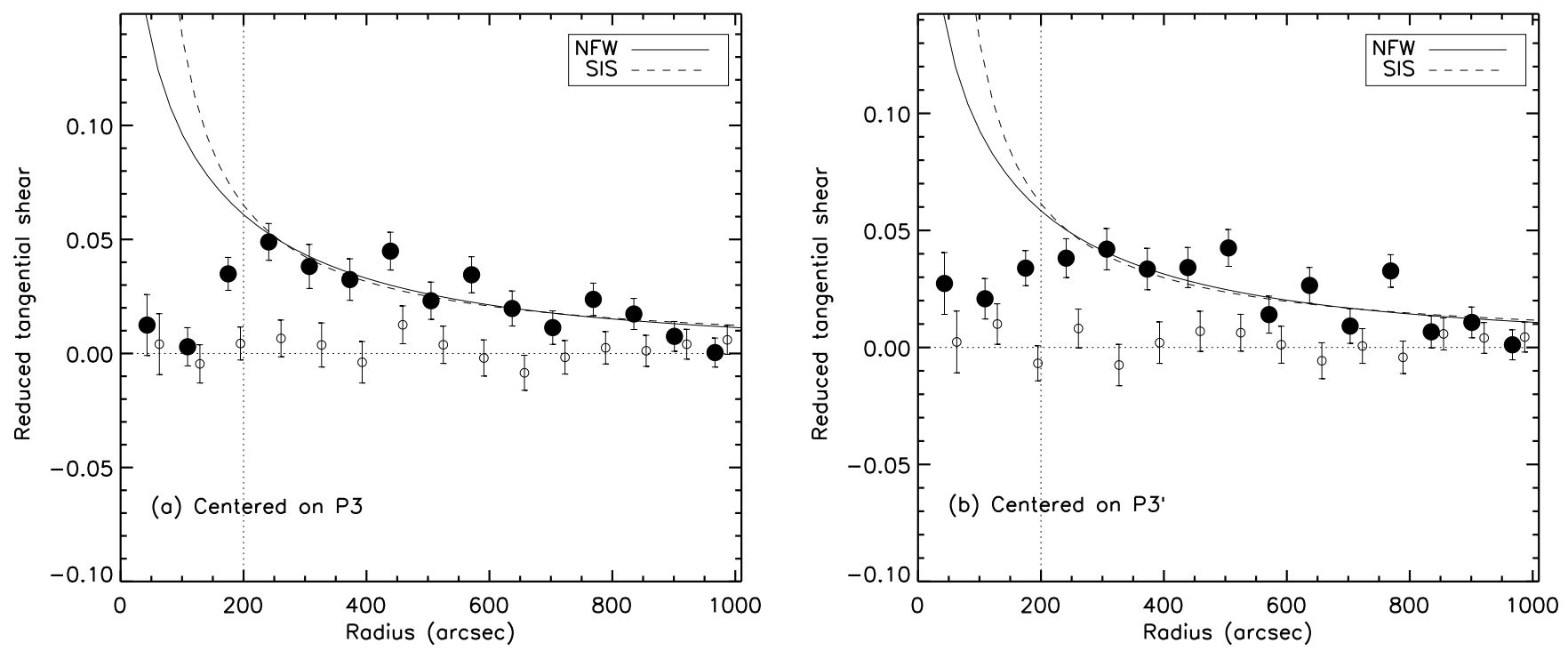

Figure 12. Tangential shear profile around P3 (a) and P3' (b). We combine the shape catalogs from the ACS and CFHT images, and applied the due redshift scaling to the CFHT shears. The relevant critical surface mass density for the plots shown here is $\Sigma_{c}=$ $3.35 \times 10^{3} M_{\odot} \mathrm{pc}^{-2}$. The filled circles represent the tangential shears around the reference points. The open circles represent our null text results obtained by rotating galaxies by $45^{\circ}$. The latter result (being consistent with zero) shows that the residual systematics in our analysis is negligible. The dotted line denotes the lower limit of the radial bin used for fitting the two model (NFW and SIS) profiles.

Table 2

Optical Luminosity of Substructure $(r<150 \mathrm{kpc})$

\begin{tabular}{|c|c|c|c|c|c|c|}
\hline Substructure & $\begin{array}{c}L_{B} \\
\left(h_{70}^{-2} 10^{11} L_{B \odot}\right)\end{array}$ & $\begin{array}{c}L_{R} \\
\left(h_{70}^{-2} 10^{11} L_{R \odot}\right)\end{array}$ & $\begin{array}{c}L_{F 814 W} \\
\left(h_{70}^{-2} 10^{11} L_{F 814 W \odot}\right)\end{array}$ & $\begin{array}{c}M / L_{B} \\
\left(h_{70} M_{\odot} / L_{B \odot}\right)\end{array}$ & $\begin{array}{c}M / L_{R} \\
\left(h_{70} M_{\odot} / L_{R \odot}\right)\end{array}$ & $\begin{array}{c}M / L_{F 814 W} \\
\left(h_{70} M_{\odot} / L_{F 814 W \odot}\right)\end{array}$ \\
\hline P1 & 1.35 & 1.52 & 2.18 & $139 \pm 32$ & $123 \pm 28$ & $86 \pm 20$ \\
\hline P2 & 3.07 & 3.31 & 4.72 & $119 \pm 9$ & $110 \pm 8$ & $77 \pm 6$ \\
\hline P3 & 0.80 & 0.98 & 1.40 & $326 \pm 43$ & $266 \pm 34$ & $186 \pm 24$ \\
\hline P3' & 0.32 & 0.38 & 0.54 & $966 \pm 97$ & $813 \pm 78$ & $572 \pm 55$ \\
\hline P4 & 3.36 & 3.83 & 4.13 & $116 \pm 9$ & $102 \pm 7$ & $94 \pm 7$ \\
\hline P5 & 2.04 & 2.53 & 2.96 & $133 \pm 19$ & $107 \pm 15$ & $92 \pm 13$ \\
\hline
\end{tabular}

Note. - We subtract the gas mass (the upper limit in Table 1 ) in the estimation of the M/L values. For the estimation of $L_{F 814 W}$, no color transformation is performed in order to ease the comparison with the C12 results.

We compare the substructure masses in Figure 13 with those obtained by J12 and C12. Our ACS weak lensing analysis of A520 provides results that are generally consistent with those from our previous WFPC2 study (J12). The central values of the masses of P1, P3, and P5 are lower in our ACS study by $\sim 20 \%, \sim 16 \%$, and $\sim 3 \%$, respectively while those of $\mathrm{P} 2$ and $\mathrm{P} 4$ are lower in our previous WFPC2 study by $\sim 5 \%$ and $\sim 14 \%$, respectively. Considering the error bars attached to these values, none of the differences causes a serious tension. Nevertheless, it is worth noting that our current ACS results give a more uniform distribution of the $\mathrm{M} / \mathrm{L}$ values (see below) for the substructures except for the dark mass region.

\subsection{Luminosity and $M / L$ Estimation}

M07 and J12 estimated the rest-frame $B$-band luminosity by selecting cluster galaxies using the CFHT $g-r$ color in conjunction with the spectroscopic catalogs. In this paper, we update the luminosities of A520 using the ACS data. The availability of three filters and the improved photometry thanks to high-resolution imaging are

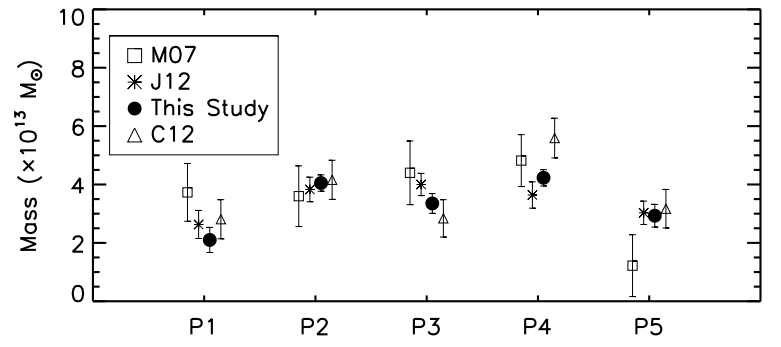

Figure 13. Mass comparison among four different studies. We compare aperture masses from M07, J12, C12, and this study. The two largest differences between this study and C12 are found for the mass estimates of $\mathrm{P} 3$ and $\mathrm{P} 4$. C12 give a lower value for P3 and a higher value for $\mathrm{P} 4$. We are able to reproduce this trend when we repeat our weak lensing analysis without performing any CTI correction (see also Figure 8). Not compared in this plot is the substructure mass of $\mathrm{P}^{\prime}$, which is not identified by $\mathrm{C} 12$. 


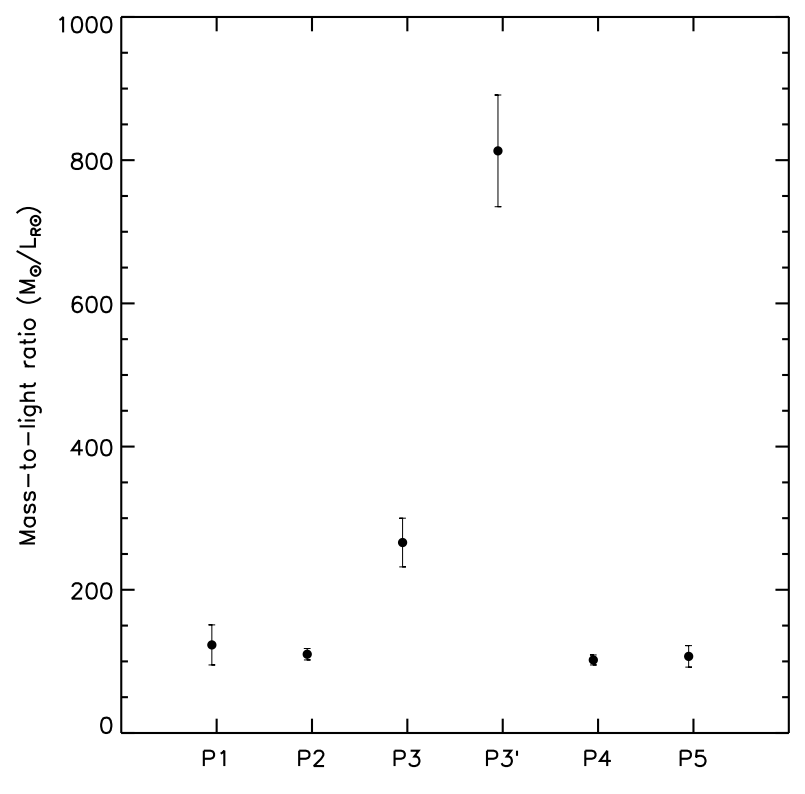

Figure 14. Mass-to-light ratio comparison among the different substructures in A520. The M/L values of the "normal" peaks (P1, $\mathrm{P} 2, \mathrm{P} 4$, and P5) are consistent with one another with small scatters around their mean $\sim 112 M_{\odot} / L_{R \odot}$. However, the dark peaks (P3 and $\mathrm{P} 3^{\prime}$ ) have considerably higher $\mathrm{M} / \mathrm{L}$ values.

expected to improve the accuracy in the A520 luminosity estimation. We define the cluster galaxies as the objects whose F606W-F814W and F435W-F606W colors are consistent with those of the spectroscopic members.

For easy comparison, we adopt the same quadrilateral boundary shown in Figure 1 of C12. We discard the object if the spectroscopic redshift is known and is not within the cluster redshift range. Also, stars are identified by comparing the light profile with that of the model PSF. The F814W filter is close to the rest frame $R$ filter at $z=0.2$, and we establish the photometric transformation by performing synthetic photometry using the spectral energy distribution (SED) templates of Kinney et al. (1996) 13 and the filter throughput curves of ACS and Johnson R; we verify that the elliptical template of Kinney et al. (1996) yields $\mathrm{F} 606 \mathrm{~W}-\mathrm{F} 814 \mathrm{~W} \simeq 0.78$ and $\mathrm{F} 435 \mathrm{~W}-\mathrm{F} 606 \mathrm{~W} \simeq 1.73$, consistent with the observed values of the A520 spectroscopic members (see Figure 1 of $\mathrm{C} 12)$.

The linear best-fit result is:

$R_{\text {rest }}=F 814 W-0.083(F 606 W-F 814 W)+0.42-D M$,

where $D M$ is the distance modulus for the cluster redshift. We summarize the rest-frame luminosity and the resulting $\mathrm{M} / \mathrm{L}$ value of the cluster substructure in Table 2 . We update the $B_{\text {rest }}$ luminosity of J12 based on the current new cluster member selection, and the results are also displayed in Table 2.

C12 did not perform any $k$-correction and converted their observed F814W magnitude into the rest-frame luminosity by simply applying the distance modulus (D.

13 We use the SEDs of the elliptical, S0, Sa, Sb, SB1, and SB2 galaxies from the Kinney-Calzetti spectral atlas.
Clowe, in private communication). We refer to this luminosity as $L_{F 814 W}$ and list our estimate also in Table 2. Our estimate of the luminosity for P3 when we ignore the color-dependence of the $k$-correction is $\sim 1.40 h_{70}^{-2} 10^{11} L_{F 814 W} \odot$, in agreement with the C12 estimate. The luminosities for the other peaks agree to $\sim 15 \%$, with the exception of $\mathrm{P} 4$, where $\mathrm{C} 12$ find a $33 \%$ lower value. However, the location of P4 in C12 is offset from the peak in the luminosity distribution, whereas the position in our mass reconstruction coincides better with the light. C12 mention that the luminosity increase by $\sim 17 \%$ when the center on the peak of the light distribution, in line with the variation we see for the other peaks. Furthermore, to facilitate the comparison with the mass reconstruction, C12 measure luminosities from the smoothed light map. Although this does not bias their mass-to-light ratios, it does reduce the luminosity measured within a fixed aperture by $\sim 5-10 \%$.

The luminosities used by C12 are nearly a factor of two higher than those of J12, but our comparison indicates that this difference is mostly due to the fact that they list results for a redder band and the lack of the $k$-correction. The Kinney et al. (1996) SED of the elliptical galaxy gives more flux in $R$ than $B$ by $\sim 35 \%$ when normalized with the SED of the Sun. In fact, as discussed above, we can reproduce the $\mathrm{C} 12$ results.

C12 argue that their selection criteria (we adopt the same criteria also in our current study) are more inclusive of blue cluster galaxy candidates than those of J12. The comparison of $L_{B} \odot$ between this study and J12 shows that the difference is small, and our our updated restframe $B$-band luminosity for P3 is only $18 \%$ higher than the $\mathrm{J} 12$ value. This is in part because the $g-r$ color selection window in J12 was broad enough to include most of the bright galaxies selected in the current study. According to the current selection, the $B$-band luminosities of P3 and P4 increase by $\sim 18 \%$ and $\sim 14 \%$, respectively. On the other hand, the $B$-band luminosities of P1, P2, and $\mathrm{P} 5$ are reduced by $\sim 12 \%, \sim 17 \%$, and $\sim 4 \%$, respectively.

For the evaluation of the mass-to-light ratios, listed in Table 2, we subtracted the upper limit of the gas mass. For the luminous substructures P1, P2, P4, and P5 we find $\mathrm{M} / \mathrm{L}$ values that are consistent with one another, yielding a mean $\mathrm{M} / \mathrm{L}$ in the $R$-band of $\sim 114 M_{\odot} / L_{R \odot}$. For the $B$-band we find a value of $\sim 131 M_{\odot} / L_{B \odot}$.

For sample of 4 clusters Hoekstra et al. (2002) found an average mass-to-light ratio of $279 \pm 21 M_{\odot} / L_{B \odot}$ (evaluated at $\mathrm{z}=0.2$, assuming passive evolution). Sheldon et al. (2009) examined a large sample of clusters observed in the Sloan Digital Sky Survey. Figure 8 in Sheldon et al. (2009) shows that on small scales the central galaxies dominate the light, resulting the mass-to-light to increase with radius, before leveling off beyond $\sim 1 \mathrm{Mpc}$. Assuming passive evolution, and taking their highest richness bin, for which the mean mass resembles that of A520 well, their results imply a value of $424 \pm 29 M_{\odot} / L_{i \odot}$ for the cluster. The average asymptotic value for the range in richness is $293 \pm 8 M_{\odot} / L_{i \odot}$.

Sheldon et al. (2009) examine the M/L as a function of distance from the BCG and their results suggest that within the inner $150 \mathrm{kpc}$, the mean M/L is about $40-50 \%$ of the asymptotic value. The values listed in Table 2 for the luminous substructures are in good agreement with 
this finding if we consider the $\mathrm{M} / \mathrm{L}$ values from Hoekstra et al. (2002). On the other hand, the M/L values of P3 and $\mathrm{P} 3^{\prime}$ are much higher. Compared to the global value $232 \pm 25 M_{\odot} / L_{B \odot}$ in $\mathrm{M} 0714$, the $\mathrm{M} / \mathrm{L}$ in $\mathrm{P} 3$ is higher by $2.4 \sigma$, but value for $\mathrm{P}^{\prime}$ is more than $7 \sigma$ higher. Our ACS weak lensing analysis therefore supports the claim of M07 and J12 for the presence of substantial dark mass in this region. In Figure 14, we compare the M/L values of the substructures in A520.

\section{DETAILED COMPARISON WITH C12}

We have compared the results from our weak lensing analysis of ACS data to the results from J12 which was based on WFPC2 observations. In this section we present a more detailed comparison with the results presented in C12. We start by noting that the overall largescale distribution of the C12 mass map is similar to our ACS result. However, there are a few differences worthy of further discussion.

The C12 mass map shows some indication of overdensity at the location of $\mathrm{P} 3^{\prime}$, but it appears more as an extension of $\mathrm{P} 4$, rather than a definite peak as seen in our mass reconstruction (Figure [5). However, our tests of the CTI correction suggest that this may be the main cause of this difference. C12 also noticed a substructure about $2^{\prime}$ north of $\mathrm{P} 4$ and labeled it as "Peak 7" (Figure 2 of $\mathrm{C} 12$ ). However, this mass peak does not appear in our mass reconstruction, although there is a weak indication of an overdensity around the location in our mass map.

The M/L values of the substructures are slightly different. The M/L value of the P3 region (150 \pm $\left.44 M_{\odot} / L_{F 814 W \odot}\right)$ from $\mathrm{C} 12$ is lower than the current value $\left(200 \pm 24 M_{\odot} / L_{F 814 W \odot}\right)$ by $\sim 20 \%$, although the two error bars marginally overlap. This happens because 1) their aperture mass of P3 is lower than ours by $\sim 15 \%$ and 2 ) their gas mass estimate $0.69 \times 10^{13} M_{\odot}$ is higher than our value $0.52 \times 10^{13} M_{\odot}$ by $\sim 33 \%$. The error bars of the aperture mass of P3 between C12 and this paper also overlap. Nevertheless, because these two results are derived from the same ACS data, it is difficult to attribute this $15 \%$ difference solely to statistical noise arising from different source galaxy selection. C12 argue that the main difference in the $\mathrm{M} / \mathrm{L}$ values between $\mathrm{C} 12$ and J12 comes from the discrepancy in the luminosity estimates, claiming that their luminosity estimate around P3 is a factor of two higher than that of J12 because their cluster member selection based on three ACS filters includes more blue cluster members that J12 might have missed. However, as already mentioned in $\$ 5.1$ we find that the factor-of-two discrepancy in luminosity arises mainly from the difference in the passband and the omission of the due $k$-correction. Using the $\mathrm{C} 12$ selection criteria, we estimate $0.80 \times 10^{11} L_{B \odot}$ in the rest-frame $B$, which is only $\sim 18 \%$ higher than the estimate of J12. Ignoring the $k$-correction, we obtain $1.40 \times 10^{11} L_{F 814 W \odot}$ in the rest-frame $\mathrm{F} 814 \mathrm{~W}$, which is in good agreement with the estimate of $\mathrm{C} 12$.

C12 presented their bootstrap resampling experiments and claimed that any substructure resembling the dark peak only happens in $\sim 2 \%$ of the total realizations. C12 speculated on the possibility that some chance alignment

\footnotetext{
14 We cannot estimate the global M/L with the current ACS data because of the field limit.
}

of the sources might have led to the detection in previous studies. However, the source number density in J12 data is significantly higher than that of M07 and Okabe \& Umetsu (2008). Furthermore, our ACS study is based on a higher source density compared to $\mathrm{C} 12$ and we confirm the presence of an overdensity. A caveat is that $\mathrm{C} 12$ focused on the location of the dark peak defined in J12, which is $\sim 1^{\prime}$ offset from the current centroid. C12 might have obtained different results if they had examined the region near $\mathrm{P} 33^{\prime}$. As stated in $\$$, our bootstrapping test shows that the dark peak $\mathrm{P} 4^{\prime}$ appears $\sim 99 \%$ of the random realizations at the $>4 \sigma$ significance.

We note that similar levels of M/L and mass discrepancy are present in other substructures as well. For instance, C12 quotes an aperture mass of $(5.59 \pm 0.68) \times$ $10^{13} M_{\odot}$ for $\mathrm{P} 4$ whereas we estimate $(4.23 \pm 0.28) \times$ $10^{13} M_{\odot}$. This $32 \%$ difference for $\mathrm{P} 4$ is in fact larger than the contrast in P3. Figure 13 shows the comparison for the rest of the substructures.

The error bars of C12 are on average a factor of two larger than ours, and here we provide detailed analysis of the discrepancy. C12 present analytic expression for estimating the $\zeta$ statistic as follows:

$$
\sigma_{\zeta}^{2}=\left(\frac{2(d \ln r) \sigma_{S N}}{1-r_{1}^{2} / r_{\max }^{2}}\right)^{2} \Sigma n_{b i n}^{-1},
$$

where $n_{\text {bin }}$ is the effective number of sources per logarithmic bin. The summation is carried out over these logarithmic bins. Note that we correct for the typographical error in $\mathrm{C} 12$, where the exponent of the $\left(1-r_{1}^{2} / r_{\max }^{2}\right)$ term should have been two (as above) not one. The equation is an approximation because 1) the integral in the $\zeta$ statistic is treated as summation, 2) the aperture mass is estimated using $\zeta_{c}$ rather than $\zeta$, and 3) the nonlinearity $g=\gamma /(1-\kappa)$ is ignored. We compare the results of this analytic error propagation with those obtained from our direct Monte-Carlo analysis and find that the approximation overestimates the errors by $70 \sim 90 \%$ for the $r=150 \mathrm{kpc}$ aperture mass given the same source density. The remaining discrepancy comes from the difference in the number density of source galaxies. The number density in $\mathrm{C} 12$ is 56 per sq. arcmin whereas it is 109 per sq. arcmin in this study. De-weighting low $\mathrm{S} / \mathrm{N}$ galaxies being considered, the rms shear of $\mathrm{C} 12$ is $\sim 30 \%$ higher than ours (\$3.3).

We find that the small source galaxy density of $\mathrm{C} 12$ is rather surprising because the typical source density in our previous weak lensing studies with HST/ACS images comfortably exceeds $\sim 100$ per sq. arcmin whenever the number of orbits per pointing is two or higher as in the current A520 data (Jee et al. 2005a; 2005b; 2006; 2007; 2011; Dawson et al. 2012). Also, even for ACS images with the depth of a single orbit, our typical number density of usable galaxies is above $\sim 70$ per sq. arcmin (Jee et al. 2011). The source number density also depends on shape measurement and image reduction method. It is possible that the C12 implementation of KSB together with the use of the square drizzling kernel gives a smaller number of usable galaxies. Nevertheless, Schrabback et al. (2010), who also use a KSB technique, still quote $\sim 76$ galaxies per sq. arcmin from their analysis of the COSMOS data, where the mean number of orbits per pointing is about one. 
Finally, we carry out a catalog-level comparison between this study and C12. C12 kindly agreed to exchange each team's shape catalogs to enable a detailed comparison. The total number of sources in C12 is 2,507 whereas it is 4,788 in this study. By looking for pairs within the distance of 0.5 , we identify 2,148 common objects between the two source catalogs.

One of the most basic sanity checks is to compare ellipticities of identical sources, and we display the results in Figure 15. Because the shape catalog provided by $\mathrm{C} 12 \mathrm{al}-$ ready includes their shear calibration, we also apply our independent (determined from image simulation) shear calibration to our source ellipticity to enable a fair comparison. Figure 15 shows that there is no major shear calibration difference. The mean slope is consistent with unity.

Having found no major systematic difference at least in global shear calibration between the two studies, we compare mass reconstruction results obtained from the common 2,148 sources. We use the FIATMAP code without the nonlinear updating $g=\gamma /(1-\kappa)$ because this may amplify the difference and hamper a fair assessment of the difference. Figure 16 shows the comparison. It is clear that our mass map created with the $\mathrm{C} 12$ shape (middle) shows the mass overdensity at P3'. This result is slightly different from Figure 2 of $\mathrm{C} 12$, where the authors perform the mass reconstruction using the combined shapes from HST and Magellan. Their HST only mass reconstruction is presented in the right panel of Figure 6 in $\mathrm{C} 12$, which shows more mass in the dark peak region and hence is more similar to our mass map created with the $\mathrm{C} 12$ shapes (middle panel of Figure 16). Our bootstrap experiment with the $\mathrm{C} 12$ weak-lensing cata$\log$ shows that a significance mass is found in the dark peak region for their weak-lensing data (Figure 7). We conclude that the $\mathrm{C} 12$ mass map supports the presence of the significant mass in the dark peak region in A520, although the slightly weaker significance might make the overdensity appear as an extension of $\mathrm{P} 4$ rather than a separate peak.

Remember that the above comparison is limited to the common sources found in both the current and C12 studies. Thus, an important question is how much the source galaxies not used by C12 (but included in our study) affect the results. In Figure 17, we present the mass reconstruction from these sources. Note that this is a completely independent mass map. The result is similar to the one presented in Figure 16. We can see the same substructures (including the dark peak) in this version. This illustrates that the faint galaxies discarded by C12 but included in the current study contains significant lensing signals. In addition, the comparison verifies that the dark peak in A520 is not caused by any potential systematic shape errors in the low $\mathrm{S} / \mathrm{N}$ galaxies.

\section{INTERPRETATION OF THE DARK PEAK}

Our ACS weak lensing analysis supports the finding of M07 and J12 of a significant amount of dark matter in A520 at a location where there are few luminous cluster galaxies. As in J12 we identify a peak, but its position has shifted (as explained in §4.3) and now coincides well with the peak in the X-ray emission. The $\mathrm{M} / \mathrm{L}$ value within the $\mathrm{r}=150 \mathrm{kpc}$ aperture of this new centroid is estimated to be $813 \pm 78 M_{\odot} / L_{R_{\odot}}$ after the gas mass is subtracted. This value is much larger than what is typically observed in clusters and either points to a pile-up of dark matter or a reduction in cluster galaxies in that region. M07 and J12 discussed a number of scenarios, and readers are referred to these two papers for details.

Here we revisit the subject of collisional dark matter as a potential origin of this dark substructure. Our motivation is not that the current ACS analysis result favors this scenario, but that it is worth investigating it given the updated centroid and substructure properties. We do note that the new location of the dark peak is in better agreement with the densest part of the X-ray emission and the morphology of this substructure is extended along the merger axis toward P2, which may support the collisional dark-matter hypothesis for the nature of the substructure.

M07 assumed a toy model, where peaks 1, 2, 4, and 5 each contributed $\sim 25 \%$ of the total mass observed in the central dark peak. Thus, the model assumes that the chance of dark matter scatter per particle during this encounter is 1 in 4 or

$$
\tau=\frac{\sigma_{D M}}{m_{D M}} \Sigma_{M} \approx 0.25,
$$

where $\Sigma_{M}$ is the effective scattering depth viewed by a particle moving along the merger axis. M07 used the mass of P3 to estimate this effective depth, and obtained $\sigma_{D M} / m_{D M} \sim 3.8 \pm 1.1 \mathrm{~cm}^{2} \mathrm{~g}^{-1}$, which is about $4 \sigma$ higher than the $\sigma_{D M} / m_{D M}<1 \mathrm{~cm}^{2} \mathrm{~g}^{-1}$ constraint from the Bullet Cluster (Markevitch et al. 2003; Randall et al. 2008).

In this paper, we revise the M07 model as follows. We decompose the mass of the dark peak into the contributions from the $\mathrm{P} 2$ and $\mathrm{P} 4$ halos, the gas mass, the dark matter associated with the cluster galaxies found within the $r=150 \mathrm{kpc}$ aperture, and the excess dark matter due to self-interaction. On the other hand, M07 considered the possibility that the entire mass of the dark peak originates from self-interaction.

Our ACS mass reconstruction indicates that the overall pre-merger cluster mass distribution might be approximately bimodal, dominated by two massive halos (P2 and $\mathrm{P} 4$ ) when we hypothesize that the dark peak in the center is produced after the collision. We estimate the contribution from the wings of these two halos by assuming an NFW profile with a scale radius of $100 \mathrm{kpc}$. The second parameters of the NFW model are determined by the lensing masses within the $r=150 \mathrm{kpc}$ aperture centered on each halo. The total contribution to the $\mathrm{P} 3^{\prime}$ mass is determined to be $\sim 1.4 \times 10^{13} M_{\odot}$ with $\mathrm{P} 2$ and $\mathrm{P} 4$ providing $\sim 0.2 \times 10^{13} M_{\odot}$ and $\sim 1.2 \times 10^{13} M_{\odot}$, respectively. Using the Cauchy-Schwarts inequality, we obtain a generous upper limit of $0.85 \times 10^{13} M_{\odot}$ for the plasma mass within the $r=150 \mathrm{kpc}$ radius of $\mathrm{P} 3^{\prime}$. The $R$-band luminosity of $0.38 \times 10^{11} L_{R \odot}$ is converted to $0.43 \times 10^{13} M_{\odot}$ using the average $\mathrm{M} / \mathrm{L}$ of the rest of the subclusters, and we assume this to represent the dark matter mass associated with the few cluster galaxies around the region. Then, the net excess mass attributed to the collisional deposit becomes $\sim 1.26 \times 10^{13} M_{\odot}$.

Now the most uncertain part of this scenario is how this excess dark matter is contributed by the subclusters of A520, and this dominates our uncertainty in the estima- 

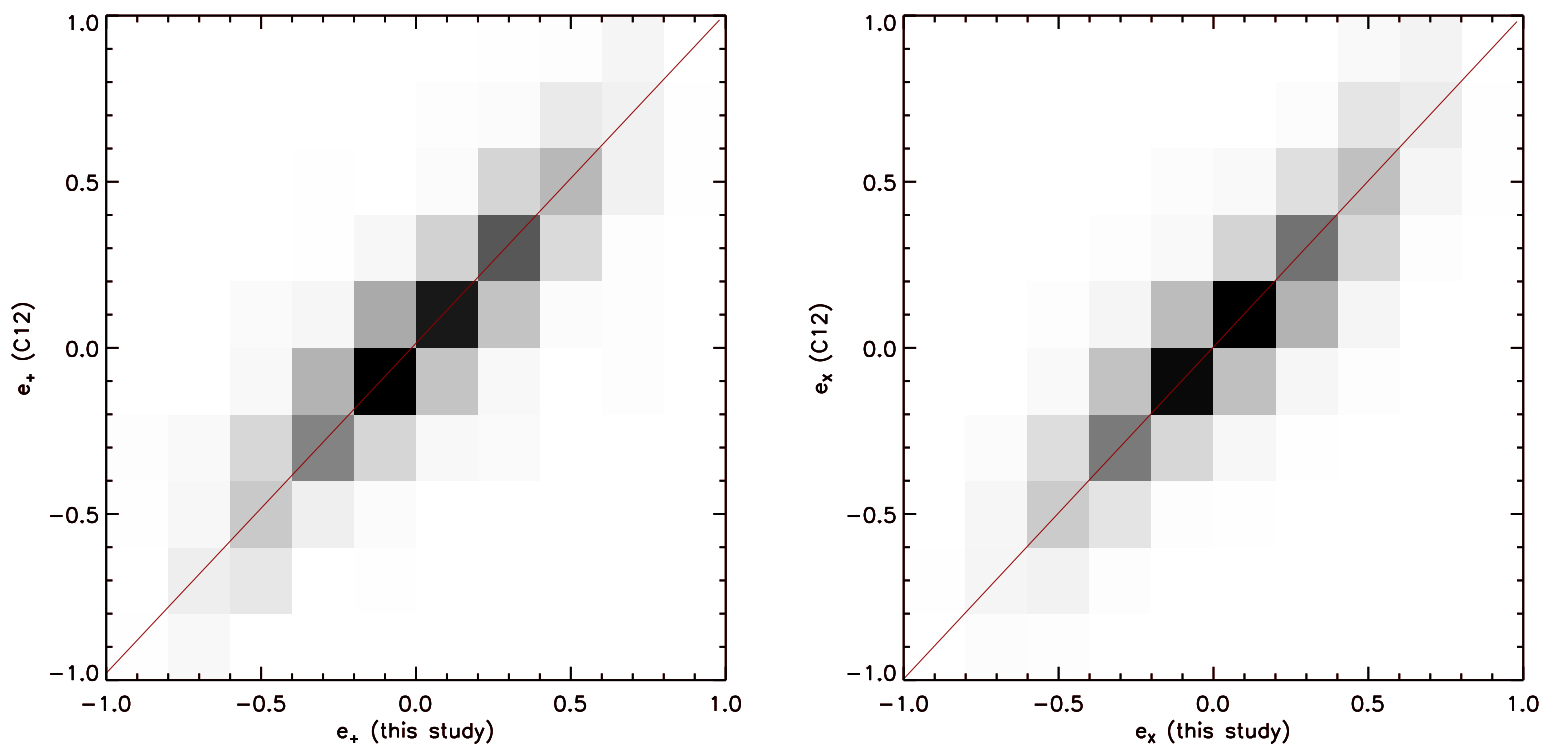

Figure 15. Shear measurement comparison between this study and C12. Shear calibration is applied. A total of 2,148 sources are common to both shape catalogs. The comparison provides basic sanity checks (e.g., systematic difference in shear calibration). The red solid line is a fit to the data, and we found that the mean slope is consistent with unity.

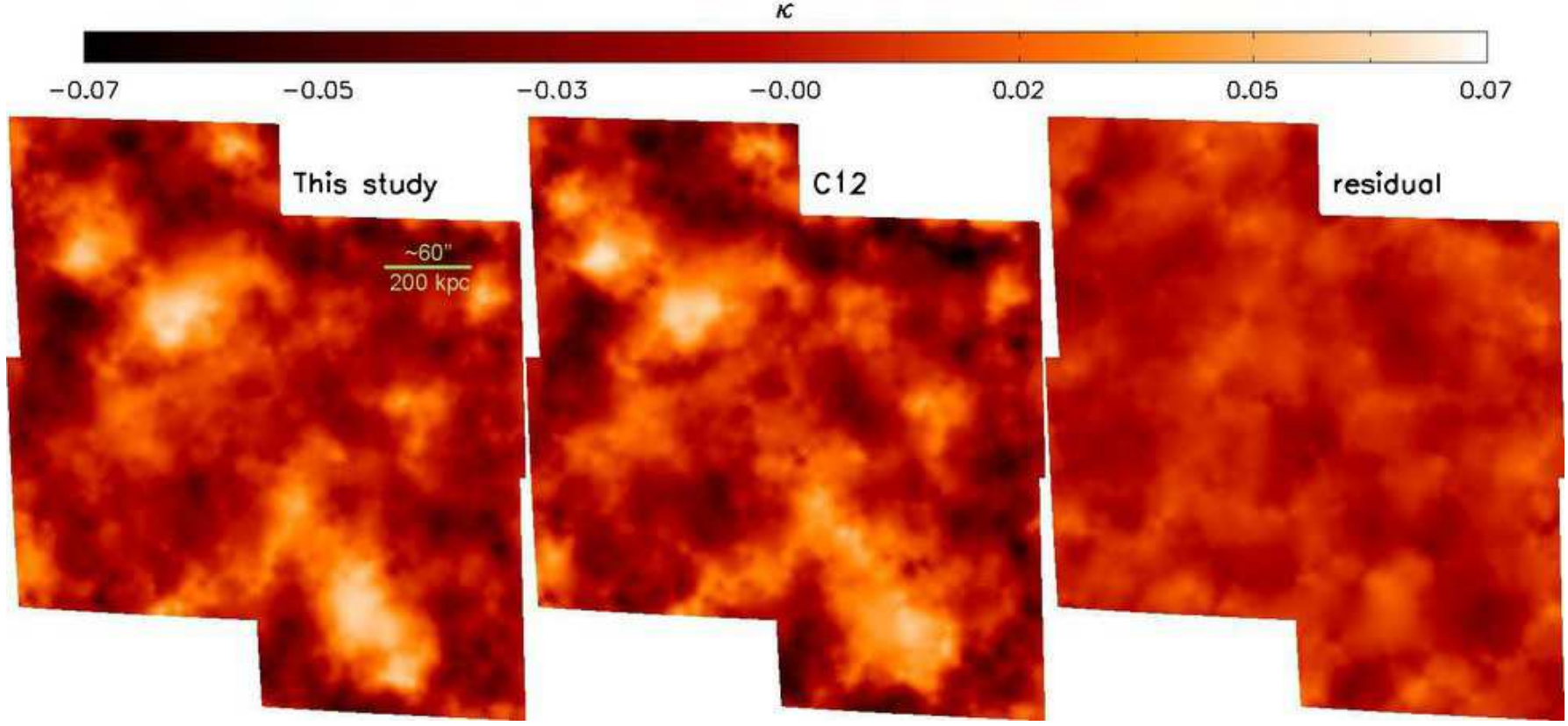

Figure 16. Direct comparison of our mass reconstruction with the C12 result. The mass reconstruction is performed with FIATMAP using the common 2,148 sources. Note that this number is more than a factor of two smaller than the total number of sources 4,788 in our study. The mass overdensity at $\mathrm{P} 3^{\prime}$ is seen in both mass maps.

tion of the collisional cross-section. If we assume P2 and $\mathrm{P} 4$ contribute equally to this excess mass through dark matter self-interaction, the mass loss fraction for each substructure is $\sim 13 \%$. The lower limit of the scattering depth $\Sigma_{M}$ is the surface mass density of P2 or P4 before the mass loss (we assume spherical symmetry). In this case, we obtain $\Sigma_{M}=0.138 \pm 0.009 \mathrm{~g} \mathrm{~cm}^{-2}$ by averaging the surface mass density of $\mathrm{P} 2$ and $\mathrm{P} 4$ and multiplying the result by 1.13 . Then, from the scattering probability of $\sim 13 \%$ we estimate $\sigma_{D M} / m_{D M} \approx 0.13 /(0.138 \pm$
$0.009) \mathrm{cm}^{2} \mathrm{~g}^{-1} \approx 0.94 \pm 0.06 \mathrm{~cm}^{2} \mathrm{~g}^{-1}$. This value does not violate the Bullet Cluster estimate $\sigma_{D M} / m_{D M} \leq$ $1 \mathrm{~cm}^{2} \mathrm{~g}^{-1}$ of Markevitch et al. (2003). Of course, the required cross-section decreases if we assume that the scattering depth is higher than the adopted value. This would happen if the dark matter particles in $\mathrm{P} 4$ have passed through more than $\mathrm{P} 2$ before it arrived at the current observed location. In fact, the location of the bow-shock feature indicates that P4 may correspond to the "bullet" of the Bullet Cluster and have experienced 

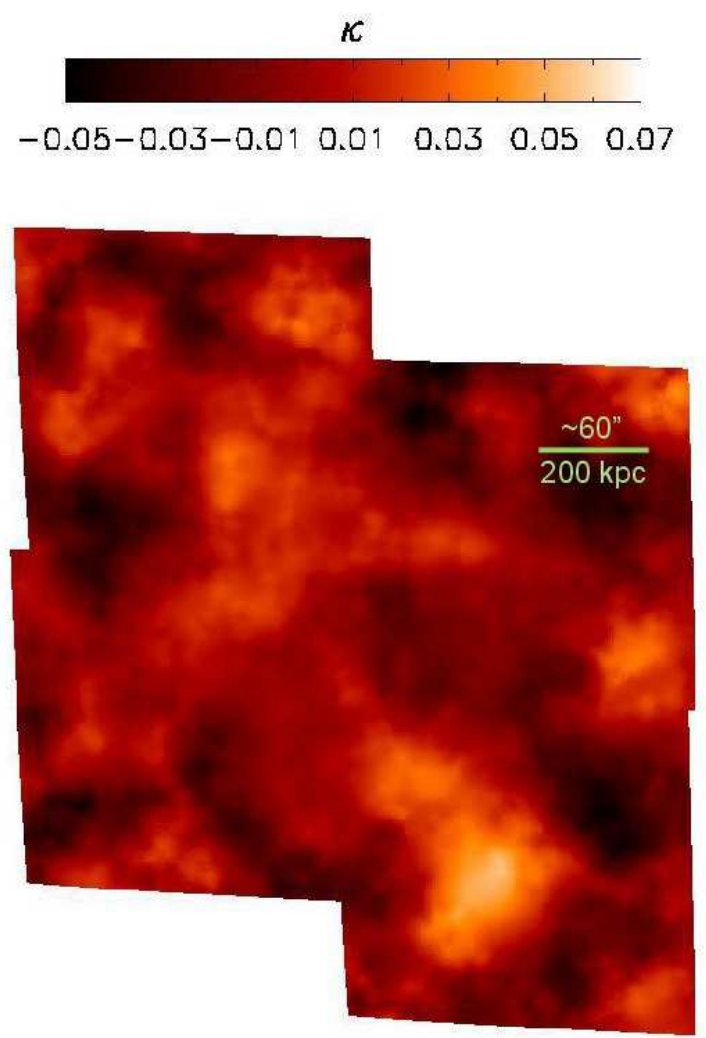

Figure 17. Mass reconstructing using the faint galaxies present in the catalog of this study but excluded by $\mathrm{C} 12$. We use FIATMAP with the remaining 2640 sources. The resulting mass map is similar to our full mass reconstruction obtained from all 4788 sources. This illustrates that the faint galaxies discarded by $\mathrm{C} 12$ but included in the current study contains significant lensing signals. In addition, the results verifies that the dark peak in A520 is not caused by potential systematic shape errors in the low $\mathrm{S} / \mathrm{N}$ galaxies.

the most acceleration, which implies that the other gravitational potential may have been deeper than that of $\mathrm{P} 2$. If we assume that $\mathrm{P} 4$ passed through $\mathrm{P} 1, \mathrm{P} 2$, and $\mathrm{P} 5$ on its way to the current location, the scattering depth increases to $\Sigma_{M}=0.302 \pm 0.019 \mathrm{~g} \mathrm{~cm}^{-2}$, which gives a cross-section $\sigma_{D M} / m_{D M} \approx 0.43 \pm 0.12 \mathrm{~cm}^{2} \mathrm{~g}^{-1}$.

Although the above constraint on the dark matter cross-section is based on simplistic assumptions of the unobserved pre-merger configuration, the result is interesting because it shows that the current observation of A520 may be explained by self-interaction of dark matter without creating any serious tension with previous values. Tighter constraints may become possible if the cluster is followed up with detailed numerical studies.

\section{CONCLUSIONS}

We have presented a re-analysis of HST / ACS images of A520. Our ACS weak lensing study confirm the presence of a region of very high mass-to-light ratio, first reported in M07 with CFHT data and subsequently supported by Obake \& Umetsu (2008) and J12 with Subaru and WFPC2 data, respectively. We are able to reproduce the results from J12 when we match the selection of our ACS weak lensing catalog to that of the WFPC2 analysis, but find no clear peak at the location where they reported one. Our detailed comparison suggest that this is caused by a variation in the source number density, which leads to additional systematic noise in the mass map. The ACS analysis shows less variation, owing in part to the overall higher number density, and thus should be more reliable.

The analysis presented here indicates a peak that is shifted by $\sim 1^{\prime}$ compared to J12. Its position now coincides well with the location of the peak of the X-ray emission. Our mass reconstruction compares well with that of $\mathrm{C} 12$, although we identify a number of differences. In particular, C12 do not identify such a clear peak, although we note an extension of $\mathrm{P} 4$ in their map towards P3'. A comparison of CTI correction algorithms, including one used by $\mathrm{C} 12$, suggest that the density contrast at the location of P3' is affected by CTI (see Figure 8). We use the latest algorithm from Ubeda \& Anderson (2012) which performs best as demonstrated in Figure 1. Note that this CTI correction method was not available to C12.

Our shape measurement analysis is able to reach a source number density of $109 \mathrm{arcmin}^{-2}$, which is considerably higher than the $\sim 56 \operatorname{arcmin}^{-2}$ used by C12. This may be explained by differences in the reduction of ACS data and how measurements in the different filters are combined. The three-filter ACS data allow for an improved membership determination which increases the luminosity by $\sim 16 \%$ compared to J12. We find that our luminosity estimates are consistent with C12 when we compare to the same band. The mass-to-light ratios (after subtracting the X-ray gas mass) of the dark peak using the old and new centroids are $285 \pm 34 M_{\odot} / L_{R \odot}$ and $813 \pm 78 M_{\odot} / L_{R \odot}$, respectively (in the rest-frame $B$-band, $349 \pm 43 M_{\odot} / L_{B \odot}$ and $966 \pm 97 M_{\odot} / L_{B \odot}$, respectively). Our $\chi^{2}$ test shows that the constant massto-light ratio hypothesis is rejected at least at the $\sim 6 \sigma$ level. The mass-to-light ratio is therefore much higher than is typically observed in clusters and could be due to a reduction in cluster galaxies or an increase in the amount of dark matter in that region. Although we still cannot single out a scenario that explains the observations, we revisit the case of collisional dark matter. With the updated substructure properties and consideration of other physical factors for the contribution to the dark peak mass, we find that the net excess mass of the dark peak region can be explained with a more conventional range of dark matter self-interacting crosssection $\sigma_{D M} / m_{D M} \approx 0.43-0.94 \mathrm{~cm}^{2} \mathrm{~g}^{-1}$, where the uncertainty is dominated by unknown scattering depth along the merger axis. This range is consistent with the results obtained from the Bullet Cluster. Detailed numerical simulations must be carried out to draw more physically meaningful constraints from the current A520 observation. Nevertheless, our analytic study hints at the possibility that A520 can be used to investigate the lower limit of the self-interacting dark matter.

We thank D. Clowe for some useful discussions and agreeing to exchange weak-lensing catalogs. M. J. acknowledges support from the National Science Foundation under Grant No. PHYS-1066293 and the hospitality of the Aspen Center for Physics. H. H. ackowledges support from NWO VIDI grant number 639.042.814. This research was supported in part by the National Science 
Foundation under Grant No. NSF PHY05-51164 and NSF PHY11-25915 to KITP. AB acknowledge support from NSERC Canada through the Discovery Grant program.

\section{REFERENCES}

Anderson, J., \& Bedin, L. R. 2010, PASP, 122, 1035

Beckwith, S. V. W., Stiavelli, M., Koekemoer, A. M., et al. 2006, AJ, 132, 1729

Bernstein, G. M. 2010, MNRAS, 406, 2793

Bradač, M., Allen, S. W., Treu, T., et al. 2008, ApJ, 687, 959

Clowe, D., Luppino, G. A., Kaiser, N., \& Gioia, I. M. 2000, ApJ, 539,540

Clowe, D., Bradač, M., Gonzalez, A. H., et al. 2006, ApJ, 648, L109

Clowe, D., Markevitch, M., Bradač, M., et al. 2012, ApJ, 758, 128

Coe, D., Benítez, N., Sánchez, S. F., Jee, M., Bouwens, R., \& Ford, H. 2006, AJ, 132, 926

Dawson, W. A., Wittman, D., Jee, M. J., et al. 2012, ApJ, 747, L42

Dawson, W. A. 2012, arXiv:1210.0014

Duffy, A. R., Schaye, J., Kay, S. T., \& Dalla Vecchia, C. 2008, MNRAS, 390, L64

Fahlman,G.,Kaiser,N.,Squires,G.\& Woods,D. 1994,ApJ, 437, 56

Fischer, P., \& Tyson, J. A. 1997, AJ, 114, 14

Giavalisco, M., et al. 2004, ApJ, 600, L93

Hack, W., Busko, I., \& Jedrzejewski, R. 2003, ASP

Conf. Ser. 295: Astronomical Data Analysis Software and Systems XII, 295, 453

Hoekstra, H., Franx, M., Kuijken, K., \& van Dokkum, P. G. 2002, MNRAS, 333, 911

Hoekstra, H. 2003, MNRAS, 339, 1155

Hoekstra, H., Donahue, M., Conselice, C. J., McNamara, B. R., \& Voit, G. M. 2011, ApJ, 726, 48
Jee, M. J., Blakeslee, J. P., Sirianni, M., Martel, A. R., White, R. L., \& Ford, H. C. 2007a, PASP, 119, 1403

Jee, M. J., et al. 2007b, ApJ, 661, 728

Jee, M. J., et al. 2009, ApJ, 704, 672

Jee, M. J., et al. 2011, ApJ, 737, 59

Jee, M. J., Mahdavi, A., Hoekstra, H., et al. 2012, ApJ, 747, 96

Jee, M. J., Hughes, J. P., Menanteau, F., et al. 2013 arXiv:1309.5097

Kaiser, N. \& Squires, G. 1993, ApJ, 404, 441

Kaiser, N., Squires, G., \& Broadhurst, T. 1995,ApJ, 449, 460

Kinney, A. L., Calzetti, D., Bohlin, R. C., et al. 1996, ApJ, 467, 38

Koekemoer, A. M., Fruchter, A. S., Hook, R. N., \& Hack, W. 2002, in The 2002 HST Calibration Workshop, ed. S. Arribas, A. Koekemoer, \& B. Whitmore (Baltimore: STScI), 337

Mahdavi, A., Hoekstra, H., Babul, A., Balam, D. D., \& Capak, P. L. 2007, ApJ, 668, 806

Markevitch, M., Gonzalez, A. H., David, L., et al. 2002, ApJ, 567, L27

Markevitch, M., Gonzalez, A. H., Clowe, D., et al. 2004, ApJ, 606,819

Markevitch, M., Govoni, F., Brunetti, G., \& Jerius, D. 2005, ApJ, 627,733

Melchior, P., \& Viola, M. 2012, MNRAS, 424, 2757

Merten, J., Coe, D., Dupke, R., et al. 2011, MNRAS, 417, 333

Okabe, N., \& Umetsu, K. 2008, PASJ, 60, 345

Randall, S. W., Markevitch, M., Clowe, D., Gonzalez, A. H., \& Bradač, M. 2008, ApJ, 679, 1173

Refregier, A., Kacprzak, T., Amara, A., Bridle, S., \& Rowe, B. 2012, MNRAS, 425, 1951

Schrabback, T., Hartlap, J., Joachimi, B., et al. 2010, A\&A, 516, A63

Seitz, S., Schneider, P., \& Bartelmann, M. 1998, A\&A, 337, 325

Sheldon, E. S., Johnston, D. E., Masjedi, M., et al. 2009, ApJ, 703,2232

Soucail, G. 2012, A\&A, 540, A61

Springel, V., \& Farrar, G. R. 2007, MNRAS, 380, 911

Ubeda, L \& Anderson, J. 2012, Instrument Science Report, ACS 2012-03 (Baltimore: STScI) 